Article

\title{
An Adaptive Decomposition Approach with Dipole Aggregation Model for Polarimetric SAR Data
}

\author{
Zezhong Wang ${ }^{(D)}$, Qiming Zeng *(D) and Jian Jiao \\ Institute of Remote Sensing and Geographic Information System, School of Earth and Space Science, \\ Peking University, Beijing 100871, China; zezhong_wang@pku.edu.cn (Z.W.); jiaojian@pku.edu.cn (J.J.) \\ * Correspondence: qmzeng@pku.edu.cn
}

Citation: Wang, Z.; Zeng, Q.; Jiao, J. An Adaptive Decomposition Approach with Dipole Aggregation Model for Polarimetric SAR Data. Remote Sens. 2021, 13, 2583. https:// doi.org/10.3390/rs13132583

Academic Editors: Luis Gómez Déniz and Raydonal Ospina

Received: 29 April 2021

Accepted: 27 June 2021

Published: 1 July 2021

Publisher's Note: MDPI stays neutral with regard to jurisdictional claims in published maps and institutional affiliations.

Copyright: (c) 2021 by the authors. Licensee MDPI, Basel, Switzerland. This article is an open access article distributed under the terms and conditions of the Creative Commons Attribution (CC BY) license (https:/ / creativecommons.org/licenses/by/ $4.0 /)$.

\begin{abstract}
Polarimetric synthetic aperture radar (PolSAR) has attracted lots of attention from remote sensing scientists because of its various advantages, e.g., all-weather, all-time, penetrating capability, and multi-polarimetry. The three-component scattering model proposed by Freeman and Durden (FDD) has bridged the data and observed target with physical scattering model, whose simplicity and practicality have advanced remote sensing applications. However, the three-component scattering model also has some disadvantages, such as negative powers and a scattering model unfitted to observed target, which can be improved by adaptive methods. In this paper, we propose a novel adaptive decomposition approach in which we established a dipole aggregation model to fit every pixel in PolSAR image to an independent volume scattering mechanism, resulting in a reduction of negative powers and an improved adaptive capability of decomposition models. Compared with existing adaptive methods, the proposed approach is fast because it does not utilize any timeconsuming algorithm of iterative optimization, is simple because it does not complicate the original three-component scattering model, and is clear for each model being fitted to explicit physical meaning, i.e., the determined adaptive parameter responds to the scattering mechanism of observed target. The simulation results indicated that this novel approach reduced the possibility of the occurrence of negative powers. The experiments on ALOS-2 and RADARSAT-2 PolSAR images showed that the increasing of adaptive parameter reflected more effective scatterers aggregating at the $45^{\circ}$ direction corresponding to high cross-polarized property, which always appeared in the $45^{\circ}$ oriented buildings. Moreover, the random volume scattering model used in the FDD could be expressed by the novel dipole aggregation model with an adaptive parameter equal to one that always appeared in the forest area.
\end{abstract}

Keywords: PolSAR; decomposition; adaptive; scattering model

\section{Introduction}

The polarimetric synthetic aperture radar (PolSAR) is a remote sensing system that combines polarimetric, synthetic aperture, and radar three key technologies [1]. Compared with the radar cross section obtained by the traditional single-polarization SAR, PolSAR contains more information of scatterers interacting with any-polarized radar by using a scattering matrix [2]. However, besides the characteristics of scatterer, e.g., orientation, shape, roughness, and dielectric constant, the scattering matrix also includes some information of the radar during transmission, propagation, and reception [3]. Thus, connecting the scattering matrix to the scatterer phenomenon is very important for the application of PolSAR data, which could be done with a physical scattering model [4]. Freeman and Durden proposed a three-component scattering model for the decomposition of PolSAR data in 1993 - the FDD [5]. It was a milestone of the application of PolSAR technology that bridged the data and observed the target with a physical scattering model whose simplicity and clarity have brought fine application results [6-10]. Subsequently, decomposition technology based on the scattering model has been widely used and continuously 
developed [11]. The higher the adaptability of the physical scattering model established in the decomposition approach, the more accurate information of the observed target that was extracted from the PolSAR image [12]. With the continuous requirement of the application and development of PolSAR sensors, the adaptability of the three-component scattering model also needs improvement [13]. For instance, if the model is only fitted to some parts of an image, the decomposition outputs corresponding to the rest of this image will be inaccurate, definitely making it difficult to apply the entire PolSAR image [14].

One way to adapt the three-component scattering model to more complex situations and to broaden the connection between PolSAR data and applications is to introduce an adaptive parameter to the scattering model. The so-called adaptive decomposition means that the scattering model contains adaptive parameters that match the data [15]. For instance, the volume scattering model in the FDD is non-adaptive because it does not including any parameters that vary with the observed data, i.e., it is assumed that the volume scattering mechanism of each observed target is exactly the same, but the fact is that different targets perform different scattering mechanisms [16]. After using adaptive parameters in the scattering model, the remaining problem is determining and explaining the adaptive parameters. The three-component scattering model in the FDD is fitted with clear physical meaning, and the FDD is a fast algorithm that can process the PolSAR image and output a concise result corresponding to scattering mechanism. Once other methods or parameters are introduced to the model, its simplicity and clarity might be reduced [11]. Some scholars such as Arii et al. [15], Chen et al. [17], and Xie et al. [18] have adopted mathematical optimization to determine the adaptive parameters while increasing the computation time for iterations. Others such as Cui et al. [19], Chen et al. [20], and Wang et al. [21] used similarity parameters, coherence parameters, and wave anisotropy as adaptive parameters while simultaneously complicating the three-component models, resulting in it being difficult for users to understand. In addition, Freeman [22], Li et al. [23], and Huang et al. [24] proposed adaptive two-component decomposition approaches by merging surface and double-bounce scattering models, but they simultaneously decreased the clarity of the decomposition results. However, few adaptive approaches have been able to keep all the FDD's properties of speed, simplicity, and clarity. In this paper, to improve the FDD while inheriting its advantages, we studied novel an adaptive approach with physical modeling, mathematical demonstration, simulation analysis, and experimental verification.

The organization of this essay is as follows. Section 2 analyzes the reasons for inadaptability of the FDD and its influence on the decomposition outputs, Section 3 proposes the dipole aggregation model with an adaptive parameter, and the adaptive decomposition with aggregation model (ADAM), Section 4 tests ADAM on space-borne PolSAR data, Section 5 discusses the advantages of ADAM, and Section 6 summarizes the main conclusions of this paper.

\section{The Principle and Problems of FDD}

The three scattering models proposed by Freeman and Durden were fitted to volume scattering from canopy, double-bounce scattering from two orthogonal planes of different material, and Bragg scattering from a rough surface [5]. They were demonstrated to work well in tropical rain forest areas [12]. In this section, we analyze why the FDD only performs well in forest areas. The FDD approach can be expressed as [12]:

$$
\langle[\boldsymbol{T}]\rangle=P_{s} \boldsymbol{T}_{s}+P_{d} \boldsymbol{T}_{d}+P_{v} \boldsymbol{T}_{v}
$$

where $\langle[\boldsymbol{T}]\rangle$ is the input of the FDD and $P_{\mathcal{S}}, P_{d}$, and $P_{v}$ are outputs corresponding to surface scattering power, double-bounce scattering power, and volume scattering power, respectively. $\langle[T]\rangle$ represents coherency matrix, also named Pauli-based covariance matrix, which is widely recognized and used for incoherent decomposition due to the well-known cloud decomposition it uses as input $[25,26]$. The FDD is an incoherent decomposition and 
assumes that each resolution unit is a multiple scattering objective, a so-called "distributed target" whose input should be a statistical average matrix, e.g., coherency matrix or covariance matrix, obtained from multilook PolSAR image [14]. For unification, in this paper, we used a coherency matrix for analysis, modeling, simulation, and experimentation; it is formulated as:

$$
\begin{aligned}
& \langle[\boldsymbol{T}]\rangle=\boldsymbol{k} \cdot \boldsymbol{k}^{* T}=\left[\begin{array}{ccc}
\left\langle T_{11}\right\rangle & \left\langle T_{12}\right\rangle & \left\langle T_{13}\right\rangle \\
\left\langle T_{12}^{*}\right\rangle & \left\langle T_{22}\right\rangle & \left\langle T_{23}\right\rangle \\
\left\langle T_{13}^{*}\right\rangle & \left\langle T_{23}^{*}\right\rangle & \left\langle T_{13}\right\rangle
\end{array}\right] \\
& k=\frac{1}{\sqrt{2}}\left[\begin{array}{lll}
S_{h h}+S_{v v} & S_{h h}-S_{v v} & 2 S_{h v}
\end{array}\right]^{T}
\end{aligned}
$$

In addition, $T_{s}, T_{d}$, and $T_{v}$ are the modeled coherency matrix corresponding to the surface scattering mechanism, double-bounce scattering mechanism, and the volume scattering mechanism, respectively. $\boldsymbol{T}_{S}$ is formulated as [12]:

$$
\mathbf{T}_{s}=\frac{1}{1+|\beta|^{2}}\left[\begin{array}{ccc}
1 & \beta^{*} & 0 \\
\beta & |\beta|^{2} & 0 \\
0 & 0 & 0
\end{array}\right]
$$

where $\beta$ is related to the local incidence angle of the radar and the relative dielectric constant of the ground. The double-bounce scattering model $\boldsymbol{T}_{d}$ is expressed as [12]:

$$
\boldsymbol{T}_{d}=\frac{1}{1+|\alpha|^{2}}\left[\begin{array}{ccc}
|\alpha|^{2} & \alpha & 0 \\
\alpha^{*} & 1 & 0 \\
0 & 0 & 0
\end{array}\right]
$$

where $\alpha$ is related to the reflectance coefficients, Fresnel reflectance coefficients, and propagation factors. The volume scattering model $\boldsymbol{T}_{v}$ is formulated as:

$$
T_{v}=\frac{1}{2}\left[\begin{array}{ccc}
1 & 0 & 0 \\
0 & \int \cos ^{2} 2 \theta p(\theta) d \theta & 0 \\
0 & 0 & \int \sin ^{2} 2 \theta p(\theta) d \theta
\end{array}\right]
$$

where $\theta$ indicates the orientation angle taken from the direction of horizontal polarization and $p(\theta)$ refers to the probability density function of random dipoles, which is always equal to $1 / 2 \pi$. Then, the volume scattering model used in the FDD can be simplified as follows [12]:

$$
\boldsymbol{T}_{v}=\frac{1}{4}\left[\begin{array}{lll}
2 & 0 & 0 \\
0 & 1 & 0 \\
0 & 0 & 1
\end{array}\right]
$$

Formula (6) shows that the volume scattering model in the FDD does not contain any adaptive parameters, i.e., assumes that the volume scattering mechanism of each observed target is exactly the same. However, different targets have different volume scattering mechanisms $[27,28]$. For example, the random dipole model in the FDD is inadaptable for a trunk structure in the vertical direction; in response to this problem, Yamaguchi et al. re-built the volume scattering model with the predomination of the vertical direction [27]. Thus, it is impossible to match all observed targets with a fixed volume scattering model, and the mismatch of them results in the inadaptability of the FDD. In order to analyze the 
impact of the model mismatch on the FDD outputs, we assumed that the coherency matrix corresponding to the actual volume scattering mechanism of the observed target is:

$$
\boldsymbol{T}_{v}{ }^{\prime}=\frac{1}{\mathrm{a}+\mathrm{b}+\mathrm{c}}\left[\begin{array}{ccc}
\mathrm{a} & 0 & 0 \\
0 & \mathrm{~b} & 0 \\
0 & 0 & \mathrm{c}
\end{array}\right]
$$

In the solution process of the FDD algorithm, $P_{v}$ can primarily be determined because all the parameters in the volume scattering model are assumed to be known. Then, $P_{S}$ and $P_{d}$ are obtained from PolSAR data by the subtracting volume component as follows:

$$
\begin{gathered}
P_{v}{ }^{\prime}=\frac{\mathrm{a}+\mathrm{b}+\mathrm{c}}{c}\left\langle T_{33}\right\rangle \\
P_{s}{ }^{\prime}+P_{d}{ }^{\prime}=\operatorname{Tr}(\langle[\boldsymbol{T}]\rangle)-P_{v}{ }^{\prime}
\end{gathered}
$$

where $\operatorname{Tr}()$ represents the trace operator and $P_{s}{ }^{\prime}, P_{d}{ }^{\prime}$, and $P_{v}{ }^{\prime}$ represent actual surface scattering power, actual double-bounce scattering power, and actual volume scattering power, respectively, corresponding to the actual volume scattering mechanism of the observed target. By contrast, the assumed volume scattering model in Formula (6) of the FDD can get a predicted results of three scattering power as:

$$
\begin{gathered}
\widehat{P}_{v}=4\left\langle T_{33}\right\rangle \\
\widehat{P}_{s}+\widehat{P}_{d}=\operatorname{Tr}(\langle[\boldsymbol{T}]\rangle)-P_{v}{ }^{\prime}
\end{gathered}
$$

Comparing the actual and predicted results shows that there might be three cases:

$$
\begin{aligned}
& \text { I. } \frac{\mathrm{a}+\mathrm{b}+\mathrm{c}}{\mathrm{c}}>4 \Longrightarrow \widehat{P}_{v}>P_{v}{ }^{\prime} \text { and } \widehat{P}_{s}+\widehat{P}_{d}<P_{s}{ }^{\prime}+P_{d}{ }^{\prime} \\
& \text { II. } \frac{\mathrm{a}+\mathrm{b}+\mathrm{c}}{\mathrm{c}}=4 \Longrightarrow \widehat{P}_{v}=P_{v}{ }^{\prime} \text { and } \widehat{P}_{s}+\widehat{P}_{d}=P_{s}{ }^{\prime}+P_{d}{ }^{\prime} \\
& \text { III. } \frac{\mathrm{a}+\mathrm{b}+\mathrm{c}}{\mathrm{c}}<4 \Longrightarrow \widehat{P}_{v}<P_{v}{ }^{\prime} \text { and } \widehat{P}_{s}+\widehat{P}_{d}>P_{s}{ }^{\prime}+P_{d}{ }^{\prime}
\end{aligned}
$$

For these three cases, only case II (12) brings a reasonable result fitted to correct physical meaning and the predicted powers of three scattering mechanisms are equal to the actual ones. Regarding case I (11), the volume scattering power $\widehat{P}_{v}$ predicted by the FDD is higher than the actual value, but the predicted powers of surface and double-bounce scattering $\left(\widehat{P}_{s}+\widehat{P}_{d}\right)$ are below the actual value. Notably, case I even leads to a prediction of a negative value for surface or double-bounce scattering powers, which violates the law of positive power. For case III (13), the volume scattering power $\widehat{P}_{v}$ predicted by the FDD is lower than the actual value, but the predicted powers of surface and double-bounce scattering $\left(\widehat{P}_{s}+\widehat{P}_{d}\right)$ are higher than the actual value. An adaptive process proposed in next section will eliminate those phenomena and keep the predicted powers equal to the actual ones.

\section{Adaptive Decomposition Approach with Aggregation Model}

\subsection{Dipole Aggregation Model}

Volume scattering is recognized as multiple scattering, which can be expressed mathematically by an integral operator [12]. A such, the dipole-based volume scattering model is the sum of scattering contributions of dipoles in different directions [29]. Under the backscattering alignment frame, the scattering matrix of horizontal dipole can be expressed as:

$$
S_{h-\text { dipole }}=\left[\begin{array}{ll}
1 & 0 \\
0 & 0
\end{array}\right]
$$


The coherency matrix corresponding to Formula (14) is:

$$
\langle[\boldsymbol{T}]\rangle=\frac{\left\langle\left[\begin{array}{lll}
S_{h h}+S_{v v} & S_{h h}-S_{v v} & 2 S_{h v}
\end{array}\right]^{T} \cdot\left[\begin{array}{lll}
S_{h h}+S_{v v} & S_{h h}-S_{v v} & 2 S_{h v}
\end{array}\right]^{*}\right\rangle}{2}
$$

Then, the sum of scattering contributions of dipoles in different directions can be expressed as:

$$
\boldsymbol{T}_{v}=\frac{1}{2}\left[\begin{array}{ccc}
1 & \int \cos 2 \theta p(\theta) d \theta & 0 \\
\int \cos 2 \theta p(\theta) d \theta & \int \cos ^{2} 2 \theta p(\theta) d \theta & 0 \\
0 & 0 & \int \sin ^{2} 2 \theta p(\theta) d \theta
\end{array}\right]
$$

where $\theta$ is the angle between the dipole and the direction of horizontal polarization. The numerical value of the element in the scattering model mainly depends on the probability distribution function $p(\theta)$ of the dipole direction. The FDD assumes that $p(\theta)$ is a uniform distribution function [12]. In this study, we used an aggregation parameter $\gamma$ in $p(\theta)$ to make Formula (16) — which refers to the aggregation degree of dipoles in the $45^{\circ}$ direction, i.e., a higher value of $\gamma$ responded to a higher value of $p\left(45^{\circ}\right)$-adaptive. When the aggregation parameter $\gamma$ takes a minimum value of 0 and a maximum value of positive infinity, the corresponding coherency matrix reaches the two extreme cases shown in Table 1.

Table 1. The structure and coherency matrix of the dipole-based model corresponding to the mini-

\begin{tabular}{|c|c|c|c|c|c|}
\hline Dipole-Based Model & $\gamma$ & \multicolumn{4}{|c|}{$T_{v}$} \\
\hline & $+\infty$ & $\frac{1}{2}$ & ( & $\begin{array}{l}0 \\
0 \\
0\end{array}$ & $\left.\begin{array}{l}0 \\
0 \\
1\end{array}\right]$ \\
\hline$H$ & 0 & $\frac{1}{2}$ & 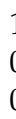 & $\begin{array}{l}0 \\
1 \\
0\end{array}$ & $\begin{array}{l}0 \\
0 \\
0\end{array}$ \\
\hline
\end{tabular}
mum and maximum value of $\gamma$.

According to Table 1, we built the aggregation model with $\gamma$ :

$$
\boldsymbol{T}_{v}=\frac{\gamma \boldsymbol{T}_{\times}+\boldsymbol{T}_{+}}{\gamma+1}
$$

where different values of $\gamma$ reflect the aggregation degrees of the dipoles. As shown in Figure 1, the aggregation model is regarded as an intermediate state of the two extreme cases in Table 1 . When $\gamma$ takes a minimum value of 0 , no dipoles present at the $45^{\circ}$ direction, but increasing $\gamma$ values leads to more dipoles moving to the $45^{\circ}$ direction. The value of the aggregation parameter $\gamma$ increases by 1 , indicating that two dipoles move to the $45^{\circ}$ direction from the horizontal and vertical directions.

After substituting the two coherency matrixes of $T_{\times}$and $T_{+}$into Formula (17), the aggregation model with $\gamma$ is formed as follows:

$$
\boldsymbol{T}_{v}=\frac{\gamma}{\gamma+1}\left[\begin{array}{ccc}
\gamma+1 & 0 & 0 \\
0 & 1 & 0 \\
0 & 0 & \gamma
\end{array}\right]
$$

where $\boldsymbol{T}_{v}$ is the coherency matrix corresponding to the adaptive dipole-based aggregation model. 


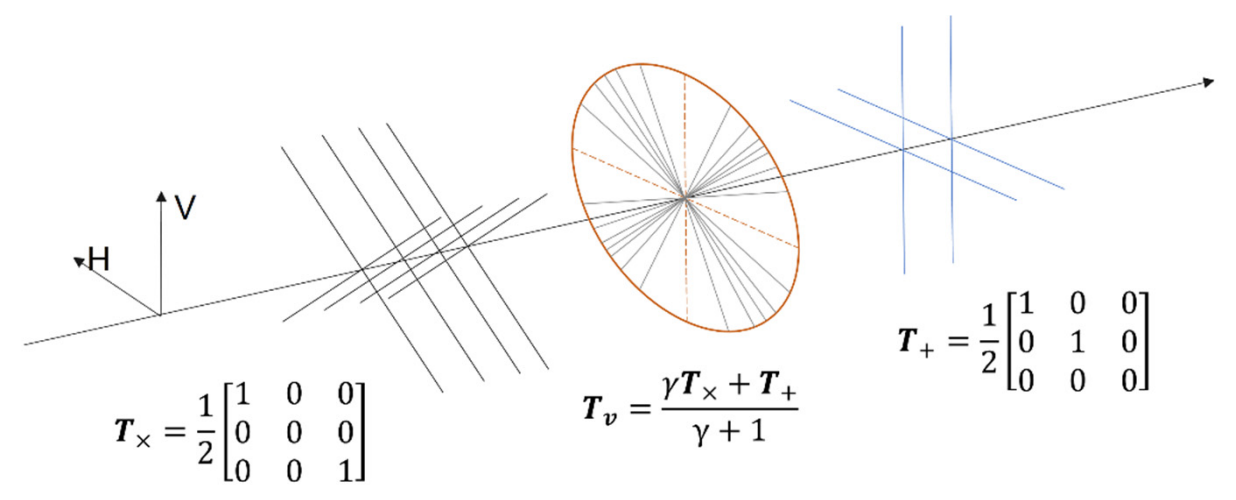

Figure 1. Dipole aggregation model with adaptive parameter $\gamma$.

As shown in Figure 2, five spectial cases were selected to demonstrate the physical meaning of the aggregation parameter $\gamma$, where the arrow points to the ascending direction of $\gamma$. Case I: when $\gamma$ was equal to $0, p\left(45^{\circ}\right)$ was equal to 0 , and $p\left(0^{\circ}\right)$ was equal to 1 , no dipoles were distributed in the $45^{\circ}$ direction in aggregation model. Case II: when $\gamma$ was equal to $1 / 2$ and $p(\theta)=\cos ^{2} \theta$, a few dipoles gathered in the $45^{\circ}$ direction in the aggregation model. Case III: when $\gamma$ was equal to 1 and $p(\theta)=1 / 2 \pi$, the dipoles were randomly distributed in the aggregation model in the same way as the volume scattering model used in the FDD [12]; additionally, when $\gamma$ was equal to $8 / 7$ and $p(\theta)=\sin \theta$, the dipoles were distributed in one predominated direction in the aggregation model in the same way as the volume scattering model proposed by Yamaguchi et al. [16]. Case IV: when $\gamma$ was equal to 2 and $p(\theta)=\sin ^{2} \theta$, most of the dipoles gathered in the $45^{\circ}$ direction and the dipoles were distributed with two predominated directions in the aggregation model in the same way as the volume scattering model proposed by Wang et al. [29]. Case V: when $\gamma$ rose to positive infinity and $p\left(45^{\circ}\right)=1$, the aggregation model reached the extreme state of dipoles being concentrated in the $45^{\circ}$ direction.

\subsection{The Algorithm of ADAM}

After replacing the volume scattering model in the FDD with adaptive aggregation model, ADAM is formed. Then, the necessary step is to determine the adaptive parameter. As discussed in Section 2, negative powers happen when the volume scattering model is unmatched with the observed target. Thus, to improve the adaptability of the FDD, the adaptive parameter in ADAM can be determined by minimizing the possibility of negative powers. Given that both $f(\gamma)$ and $g(\gamma)$ are functions of $\gamma$, the relationship between $\gamma$ and the phenomenon of negative powers can be described by the following three inequalities:

$$
\begin{gathered}
T_{11}-f(\gamma) T_{33}>0 \\
T_{22}-g(\gamma) T_{33}>0 \\
\left(T_{11}-f(\gamma) T_{33}\right)\left(T_{22}-g(\gamma) T_{33}\right)-\left|T_{12}\right|^{2}>0
\end{gathered}
$$

where the satisfaction of three inequalities can ensure non-negative powers according to the principle of the FDD [12,30]. Figure 3 shows the two functions of $f(\gamma)=1 / \gamma+1$ and $g(\gamma)=1 / \gamma$, both of which are the decreasing function of $\gamma$ so that there is a minimum value of $\gamma$ that satisfies the Formula (19) and (20). The left side of the third inequality in Formula (21) can be transformed into a quadratic polynomial related to $\gamma$; when it is equal to 0 , there are two solutions of $\gamma$, where the smaller one must be contrary to the Formula (19) and (20) but the larger one needs to meet them. Thus, the adaptive parameter in ADAM is determined as the larger solution, and Figure 4 shows the entire determination process of adaptive parameter $\gamma$. 


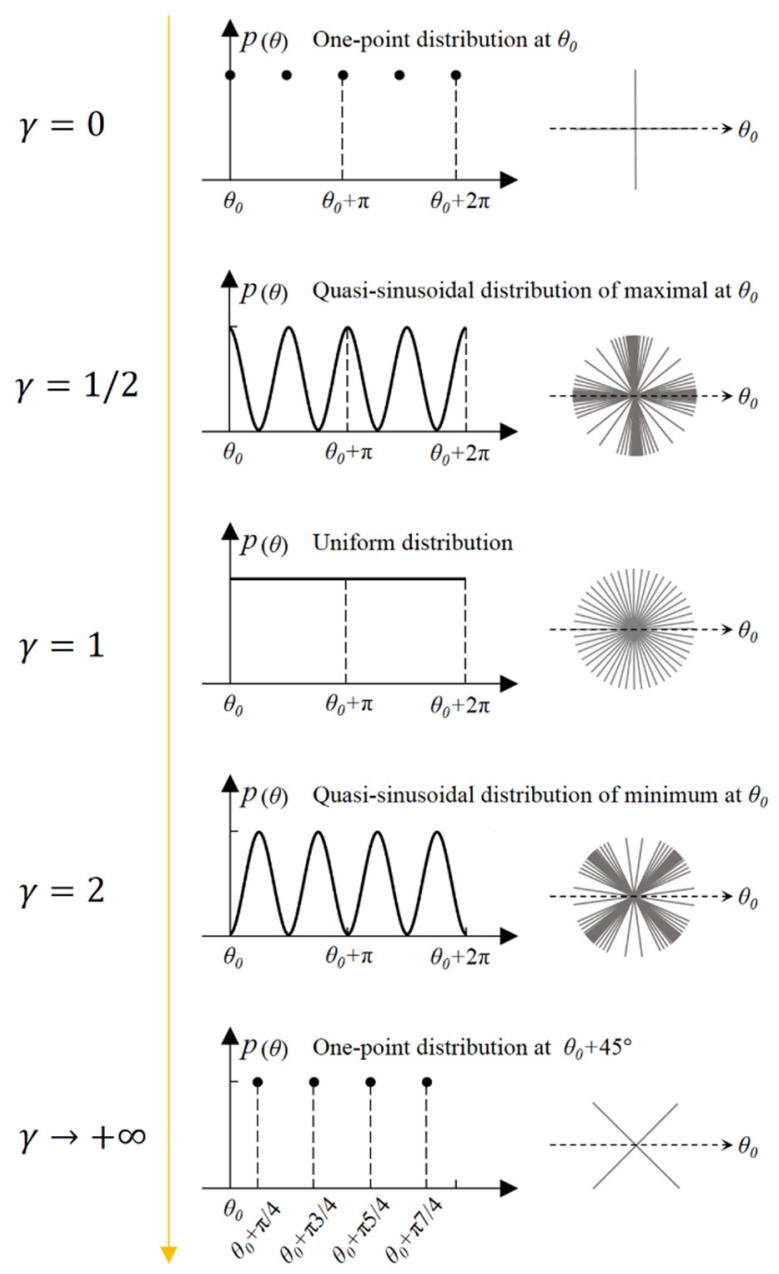

Figure 2. The physical meaning of aggregation parameter $\gamma$ with different numerical values.

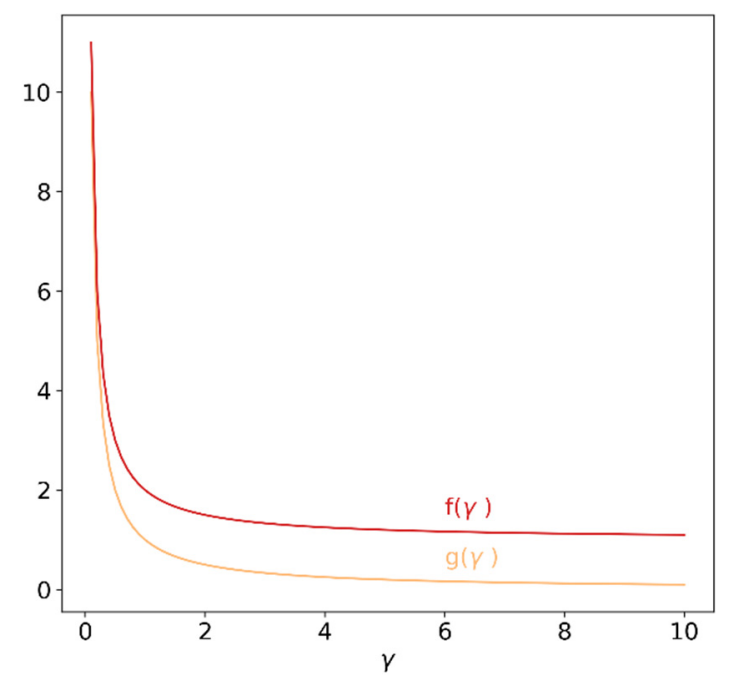

Figure 3. Diagram of $f(\gamma)$ and $g(\gamma)$ functions.

After the adaptive parameter in volume scattering model has been determined, the remaining procedure is the same as that of the FDD [1]. Here, the algorithm of ADAM was implemented with Python 3.5 and the corresponding codes created in this study were shared on GitHub (https:/ / github.com/lengmeiqing/ADAP, accessed on 29 April 2021). 


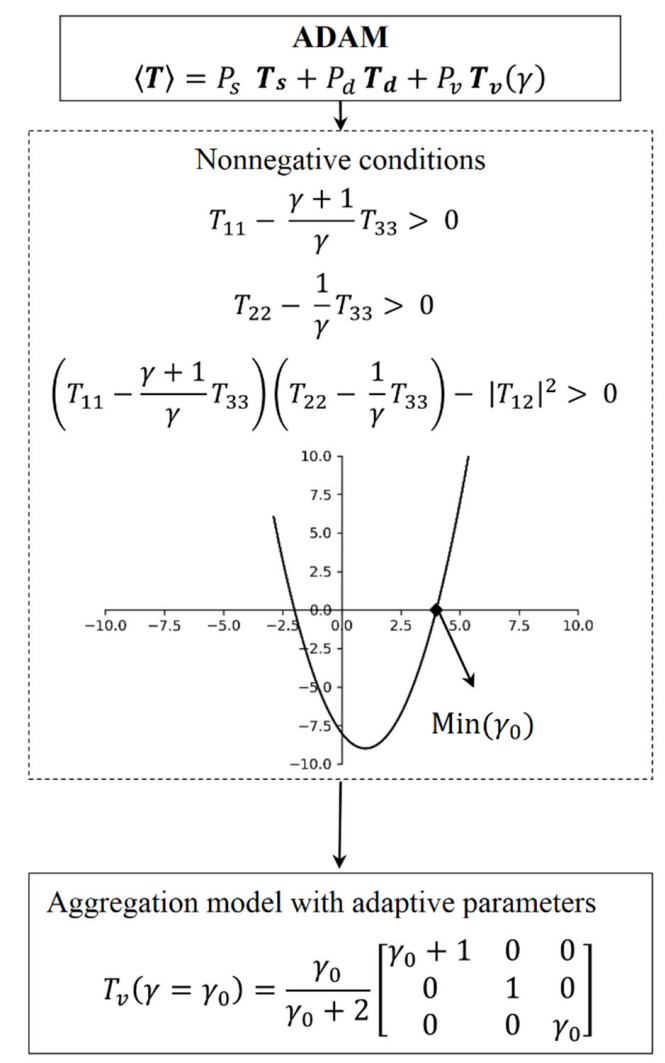

Figure 4. Determination of the adaptive parameter in the dipole aggregation model.

Figure 5 illustrates the relationship between the adaptive parameter $\gamma$ and $T_{11}$ and $T_{22}$ in the coherency matrix under the assumption that $T_{33}=1$ and $T_{33}=0$. The horizontal axis represents $T_{11}$, the color represents $T_{22}$, and the vertical axis represents the determined adaptive parameter. The variation lines in Figure 5 indicate the minimum solution of $\gamma$ that satisfies Formula (21), and each variation line of $\gamma$ starts from curve and end to horizontal line. The turning point from curve to horizontal line for each variation line responds to $T_{11}-T_{22}=1$, i.e., the value of $\gamma$ varies with $T_{11}$ when $T_{11}-T_{22}<1$ and the value of $\gamma$ changes with $T_{22}$ when $T_{11}-T_{22}>1$. For all cases of $T_{11}$ and $T_{22}$, the value of $\gamma$ above the variation line leads to non-negative powers of $P_{S}$ and $P_{d}$; otherwise, it results in negative powers. For $\gamma$ in the FDD, which is always fixed on 1 , only the cases of $T_{11}$ and $T_{22}$ above the horizontal line of $\gamma=1$ can lead to non-negative powers of $P_{s}$ and $P_{d}$. The simulation results indicated that ADAM can reduce the possibility of negative powers in the FDD.

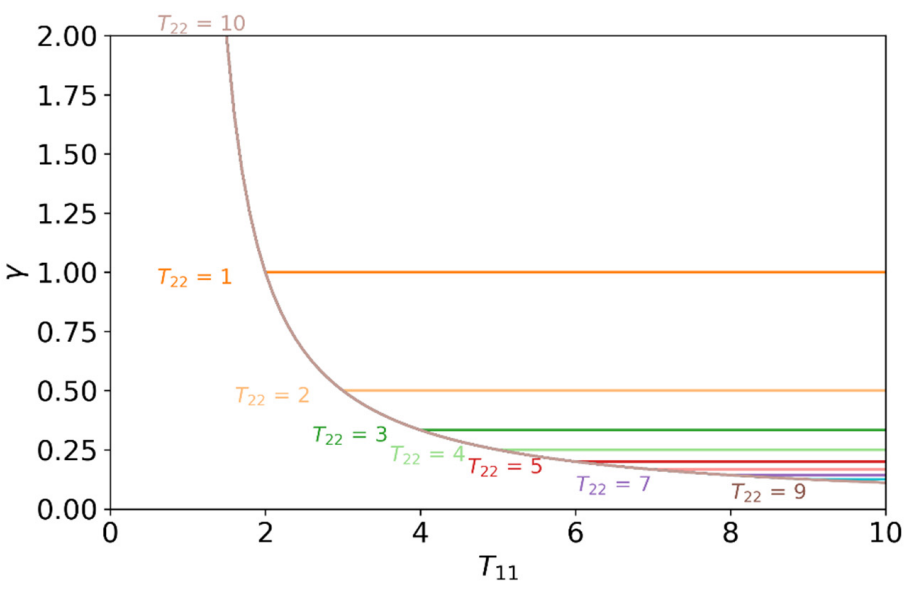

Figure 5. The simulation of the determination of $\gamma$ with $T_{11}$ and $T_{22}$. 


\section{Experiments on Space-Borne PolSAR Data}

\subsection{Experiment Scheme}

To further evaluate ADAM, we implemented it on ALOS-2 L-band space-borne PolSAR data over two experimental areas. The first experimental area is located in San Francisco, the United States $\left(37.78^{\circ} \mathrm{N}, 122.42^{\circ} \mathrm{W}\right)$, and it was imaged on August 22, 2017, by ALOS- 2 with ascending orbit, the HBQ mode, and a minimum incidence angle of $30.8^{\circ}$. The second experimental area is located in Wuhan, China $\left(10.6^{\circ} \mathrm{N}, 114.42^{\circ} \mathrm{E}\right)$, and it was imaged on January 8, 2016 by ALOS- 2 with ascending orbit, the HBQ mode, and a minimum incidence angle of $33.2^{\circ}$. The experiment scheme is shown as Figure 6 , and the experimental process is described step by step as follows:

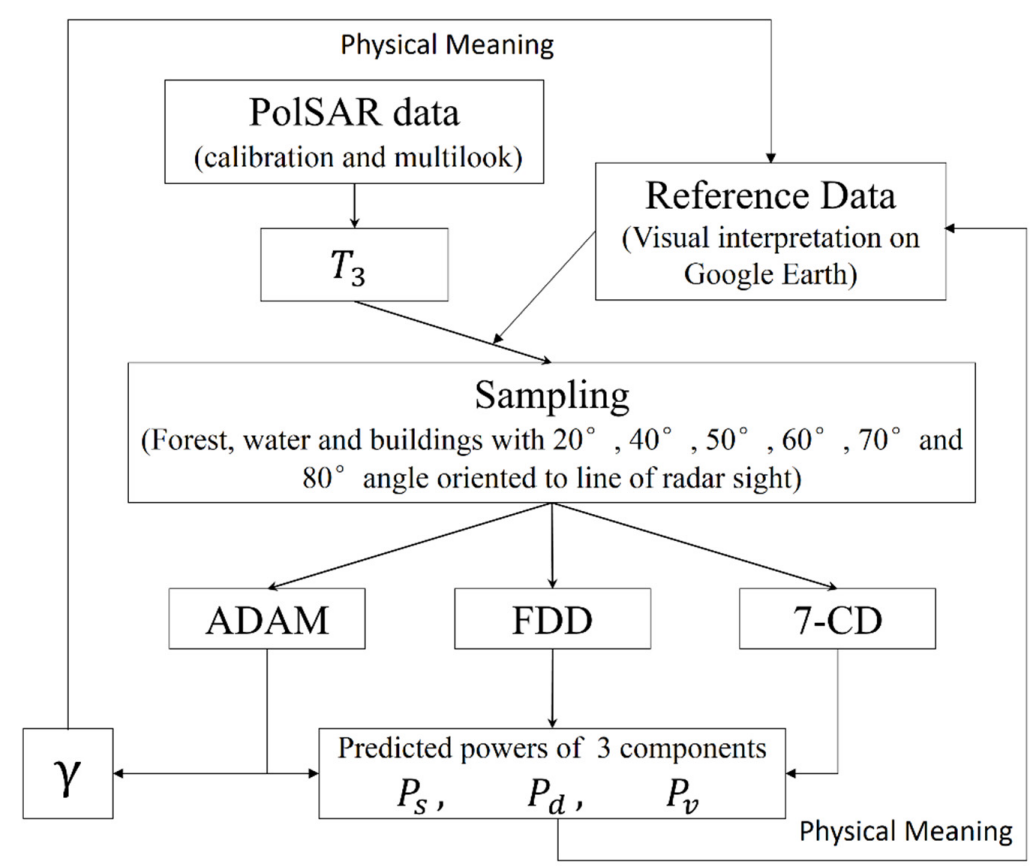

Figure 6. The experimental scheme to evaluate ADAM on space-borne PolSAR data.

Step 1 is calibration and multilook, where the ALOS-2 digital images were calibrated as:

$$
\sigma_{s l c}^{o}=10 \log _{10}\left[\left(I^{2}+Q^{2}\right)\right]+C F_{1}-A,
$$

where $C F_{1}$ and $A$ are the calibration coefficients. The $C F_{1}$ for ALOS-2 PALSAR-2 JAXA standard product was obtained as $-83 \mathrm{~dB}$, and the $A$ for SLC data was equal to $-32 \mathrm{~dB}$. All used PolSAR images were processed with a $10 \times 6$ multilook (azimuth $\times$ range) [31].

Step 2 is sampling, where the performance of targets on a PolSAR image is related to their physical properties, such as their size, shape, and material, as well as the angle of the scattering direction to the line of radar sight [32]. For example, forests, water, and buildings should present different features on a PolSAR image, but buildings also have different scattering characteristics at different orientation angles [33]. As shown in Figure 7, the orientation angle of buildings is difficult to describe due to their diverse arrangements [34]. One approach to determine the orientation angle of one building is selecting the longest wall in the building and measuring its direction representing the direction of the building [34]. However, this way might not be available for measuring the orientation angle of buildings on a medium-resolution PolSAR image. In this study, we approximately estimated the orientation of buildings by visually interpretating the result of the main street direction. As shown in Figure 8, eight types of samples performing differently with the PolSAR image were selected to analyze the ADAM method; they were 
forest, water, and buildings with $20^{\circ}, 40^{\circ}, 45^{\circ}, 50^{\circ}, 70^{\circ}$ and $80^{\circ}$ angles oriented to line of radar sight.
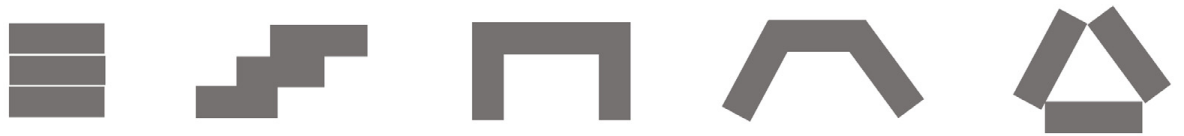

Figure 7. The different types of building arrangements.
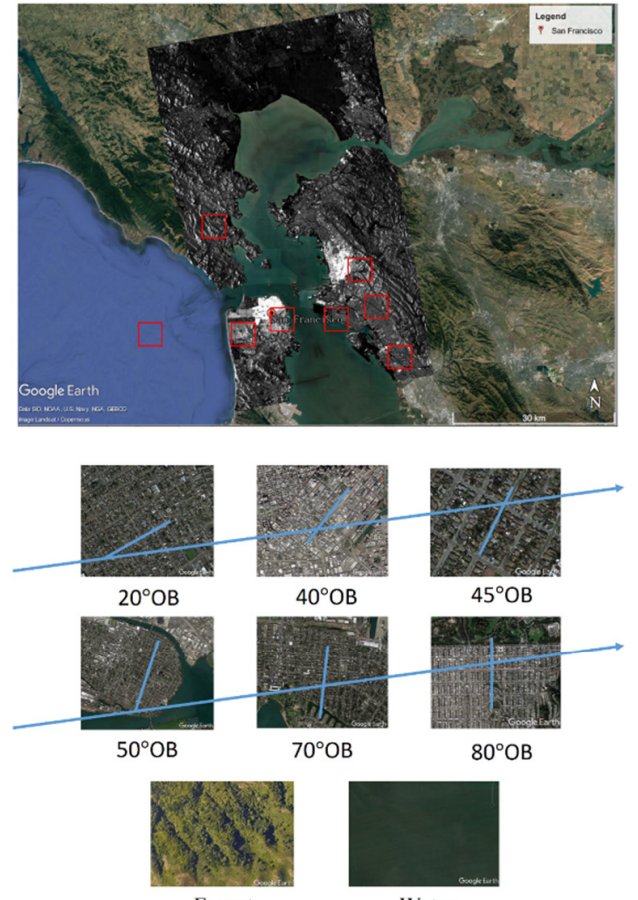

Forest

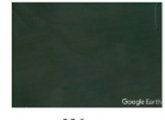

Water

(a) San Francisco, USA
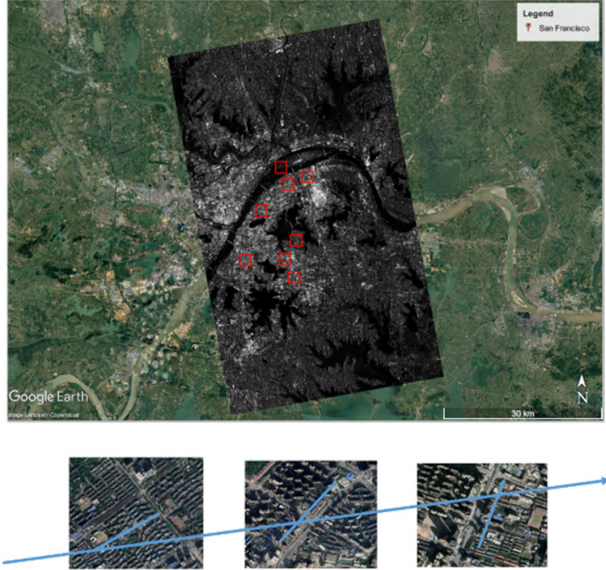

$45^{\circ} \mathrm{OB}$
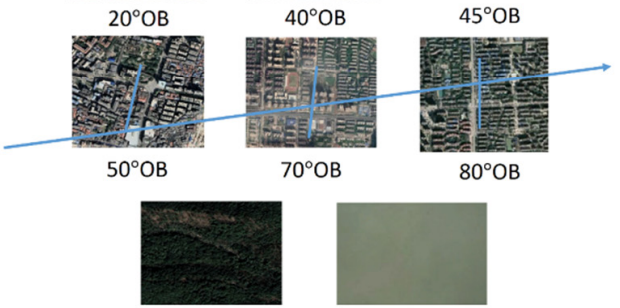

Forest

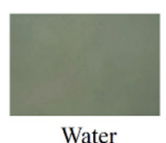

(b) Wuhan, China

Figure 8. Google Earth images and samples in San Francisco, USA (a) and Wuhan, China (b).

Step 3 is decomposition, where to assess ADAM by control experiments, we selected another two decomposition approaches for comparison. The first was the FDD since the aim of this study was to improve its adaptability, and the second was the seven-component decomposition (7CD) approach proposed by Singh et al. [35] because it represents the latest development of four-component decomposition approach, which is a well-known and widely used extension of the FDD [16,27]. In this study, all three approaches of decomposition were implemented on a PolSAR image with Python 3.5 (https: / / github. com/lengmeiqing/ADAP, accessed on 29 April 2021), and all the negative powers in outputs were maintained for analysis but not made up to zero.

Step 4 is the analysis of the physical meaning of the outputs, where the relationship between the adaptive parameter in ADAM and the property of target was analyzed by statistics and a visual map. The predicted powers of surface, double-bounce, and volume scattering by ADAM, FDD, and 7CD were compared at each sample plot.

\subsection{Interpretation of Results}

4.2.1. Physical Meaning of Aggregation Parameter

The RGB-coded images shown in Figures 9 and 10 illustrate the decomposition results of ADAM, FDD, and 7CD over the San Francisco Bay area, where a black rectangle marks an area with oriented buildings. There were some differences in the three decomposition methods especially in the marked area, as ADAM showed the red color but the FDD and 
7CD presented the green color. Red indicates the predominating scattering mechanism of double-bounce scattering, but green and blue correspond to volume scattering and surface scattering, respectively. The oriented buildings mainly contributed double-bonce scattering in the same way as the other buildings for their ground-wall structures. Thus, among the three decomposition methods, only AMAD fit the actual scattering mechanism of the observed targets. Next, we further analyzed the performance of the aggregation parameter in the results.

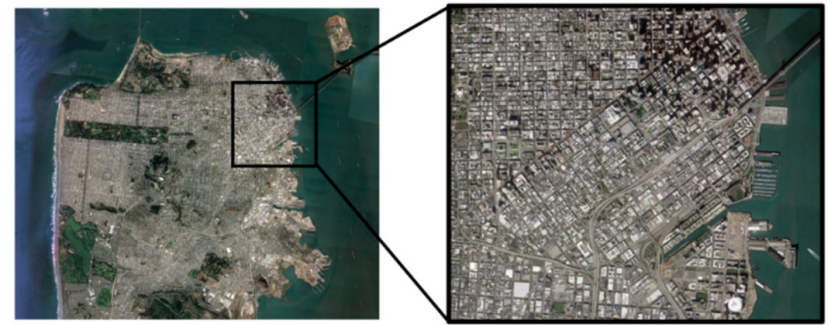

(a) High resolution satellite image over San Francisco

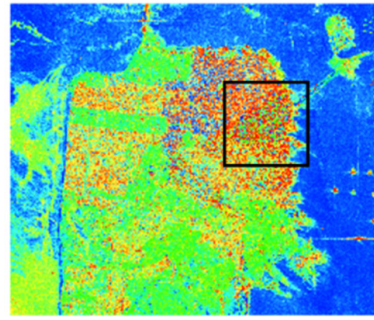

(b) ADAM

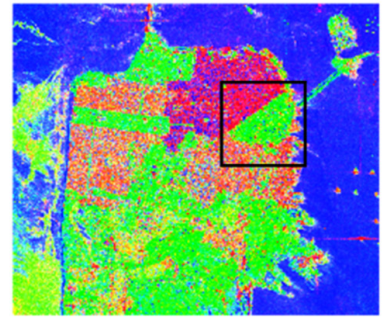

(c) FDD

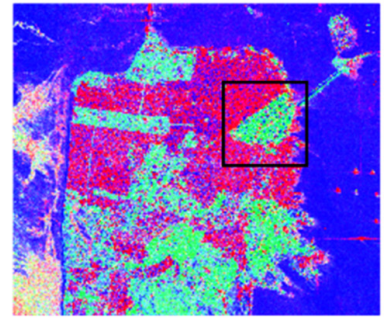

(d) $7 \mathrm{CD}$

Figure 9. RGB-coded images of the decomposition results of ADAM, FDD, and 7CD with a reference high resolution satellite image over San Francisco $\left(P_{s}\right.$-blue; $P_{d}$-red; and $P_{v}$-green).

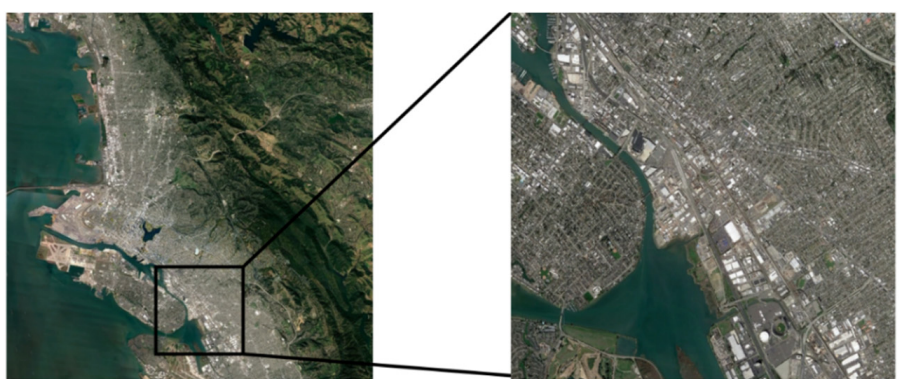

(a) High resolution satellite image over East Bay of San Francisco Bay Area

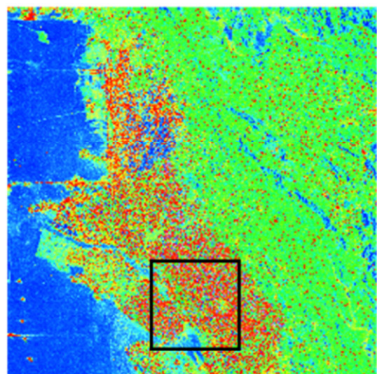

(b) ADAM

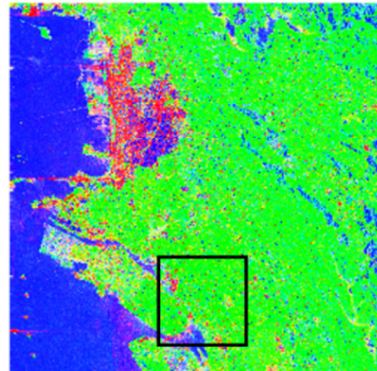

(c) FDD

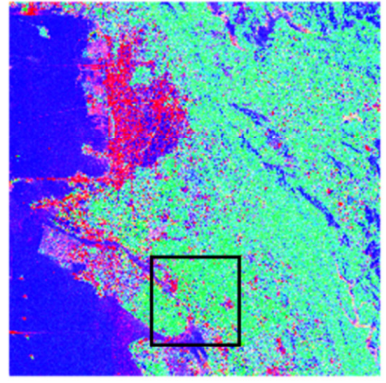

(d) $7 \mathrm{CD}$

Figure 10. RGB-coded images of the decomposition results of ADAM, FDD, and 7CD with a reference high resolution satellite image over the East Bay of the San Francisco Bay area $\left(P_{s}\right.$-blue; $P_{d}$-red; and $P_{v}$-green).

Table 2 shows the statistical results of aggregation parameter $\gamma$ corresponding to buildings, forest, and water sections on an ALOS-2 PolSAR image over San Francisco, USA. 
Before the statistical process, we used the $1.5 \mathrm{x}$ interquartile range (IQR) rule to remove the outlier values below $\mathrm{Q} 1-(1.5 \times \mathrm{IQR})$ or above $\mathrm{Q} 3+(1.5 \times \mathrm{IQR})$, where $\mathrm{Q} 1$ and $\mathrm{Q} 3$ are the first quartile $(25 \%)$ and the third quartile $(75 \%)$ of the data, respectively.

Table 2. The statistical results of the aggregation parameter $\gamma$ for 8 types of samples over San Francisco, USA.

\begin{tabular}{lcc}
\hline & \multicolumn{2}{c}{ Aggregation Parameter $\gamma$} \\
\cline { 2 - 3 } & Mean & STD \\
\hline $20^{\circ}$ oriented building & 1.07 & 0.76 \\
$40^{\circ}$ oriented building & 1.76 & 1.23 \\
$45^{\circ}$ oriented building & 2.39 & 1.67 \\
$50^{\circ}$ oriented building & 2.15 & 1.6 \\
$70^{\circ}$ oriented building & 1.01 & 0.53 \\
$80^{\circ}$ oriented building & 0.68 & 0.35 \\
Forest & 1.86 & 0.76 \\
Water & 0.42 & 0.11 \\
\hline
\end{tabular}

The mean of $\gamma$ was at the maximum for the $45^{\circ}$ oriented building and at the minimum for the water. Figure 11 shows that high values of $\gamma$ appeared around the $45^{\circ}$ oriented buildings with the blue color, and low values of $\gamma$ appeared near $0^{\circ}$ oriented buildings with the red color, i.e., a higher value of $\gamma$ appeared with the $45^{\circ}$ oriented buildings. It can be inferred that there is a potential relationship between the aggregation parameter and the scatterer's direction. The buildings were found to have more effective scatterers in the $45^{\circ}$ direction, with a high cross-scattering property fitted to aggregation model with a high value of $\gamma$ [36]. The forest area was almost blue $(\gamma>1)$, water was almost red $(\gamma<1)$, and the mean of $\gamma$ over forest was approximately equal to 2 , which was consistent with the canopy scattering model proposed by Wang [29].

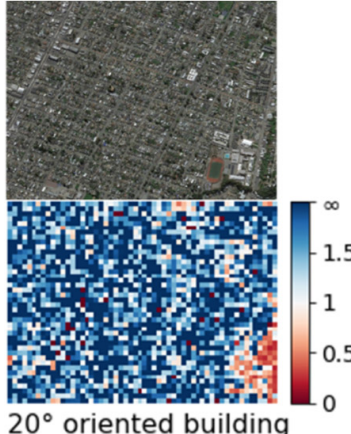

(a)

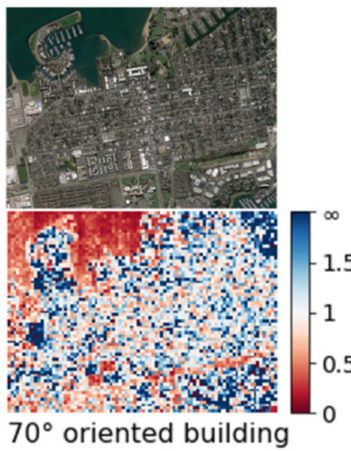

(e)

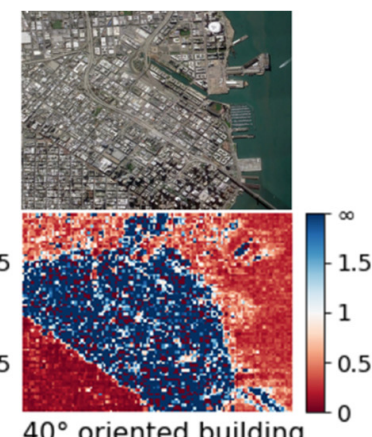

(b)

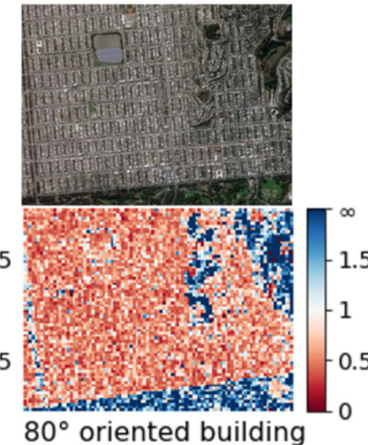

(f)

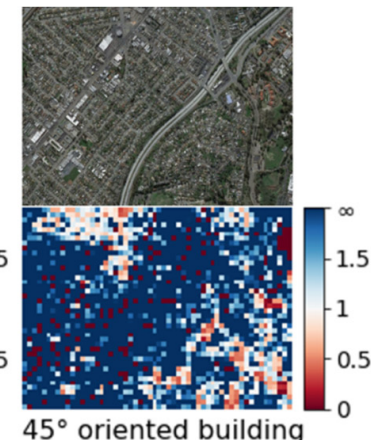

(c)

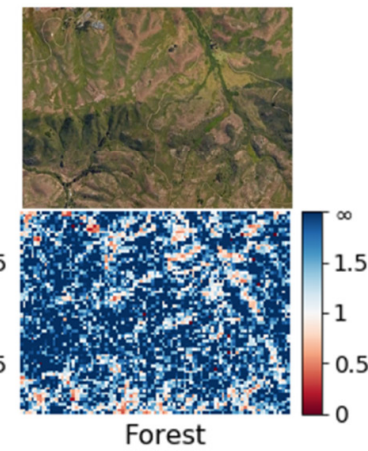

(q)

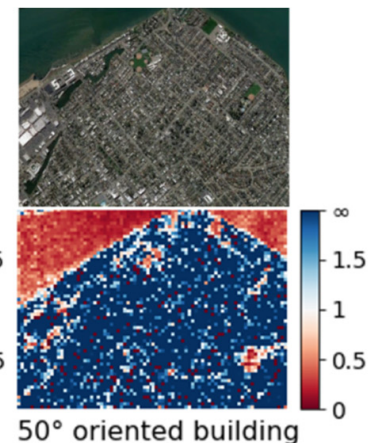

(d)

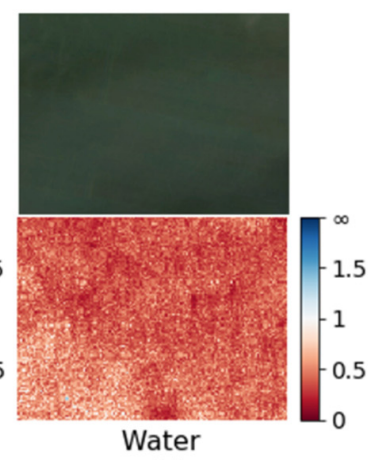

(h)

Figure 11. The determined value of aggregation parameter $\gamma$ in building samples over San Francisco, USA. 
To further analyze the physical meaning of $\gamma$, we selected more PolSAR data from an RADARSAT-2 C-band image over San Francisco to compare the performance of $\gamma$ between different land-uses. As shown in Figure 12a, the golf course, ocean, and different oriented buildings could be distinguished by values of $\gamma$ with different colors. The rectangle box with white border in Figure 11 a represents the selected samples of $5^{\circ}, 30^{\circ}, 30^{\circ}$, and $45^{\circ}$ oriented buildings; the golf course; and the ocean. Figure 12a shows the statistical results of the samples, indicating that it was easier to fit a high value of $\gamma$ with a higher mean value for $45^{\circ}$ oriented buildings than other types of samples, as well as that the reduction of orientation angle led to the decline of $\gamma$. In addition, when the aggregation parameter was adapted to 1, it corresponded to the random volume scattering model in the FDD, which fits the scattering characteristics of vegetation [12]. This case appeared in the golf course, which presented alternated colors of white and blue with a mean value of 1.09, as shown in Figure 12. The ocean was almost red and had the lowest value of $\gamma$ compared to other types of samples since it had weakest capability to change the polarization state of the radar wave [37]. Overall, the ADAM method could not only adapt to building areas with different orientations and fit them with a suitable volume scattering model but also adapt to the golf course and ocean according to their scattering mechanisms. While retaining the adaptability in vegetation area, the ADAM method improved the adaptability of three-component model to large oriented buildings and water areas.

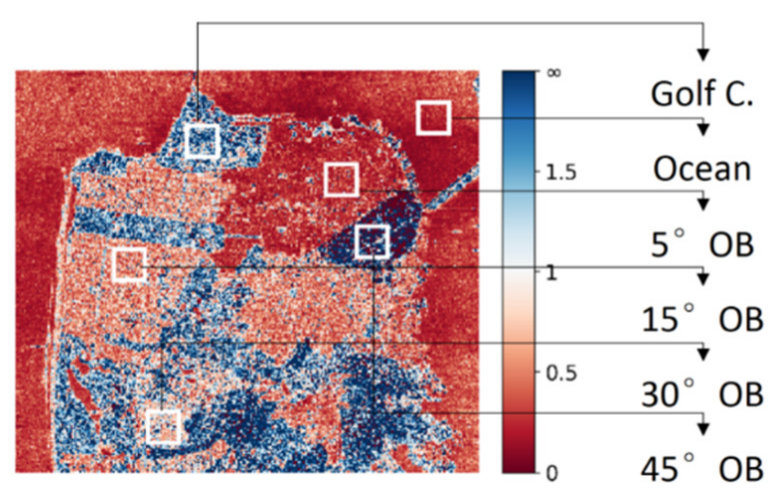

(a)

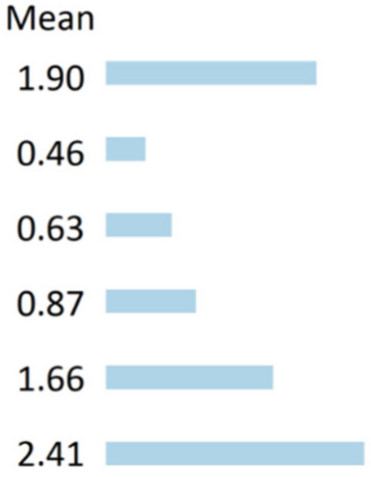

2.41

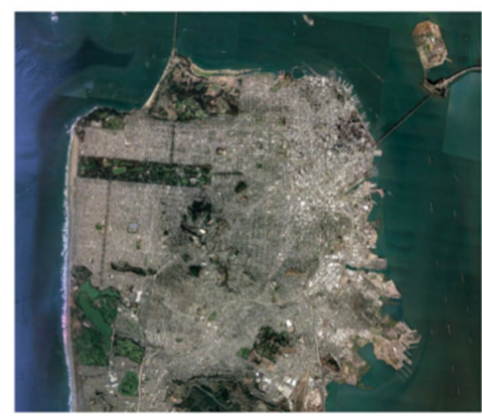

(b)

Figure 12. (a) The output map of aggregation parameter for ADAM implemented on a RADARSAT-2 C-band image over San Francisco, USA, with the statistical results of $\gamma$ of different land-use types; (b) high resolution satellite image (OB: oriented building).

\subsubsection{Comparison of ADAM, FDD and 7CD}

The comparative analysis results of Tables 3-5 show that ADAM had a better stability than the FDD for a lower statistical standard deviation (STD), which will lead to a better interpretation of scattering mechanisms for one type of target without a large feature boundary. In addition, ADAM was found to have a lower possibility of negative powers appearing in $P_{s}$ and $P_{d}$ than the FDD, as shown in Figures 13 and 14, and it showed a lower possibility of negative powers presenting in $P_{v}$ than 7CD, as shown in Figure 15. The reason that ADAM was able to mitigate the occurrence of negative powers in the FDD was that the underestimated $P_{S}$ and $P_{d}$ with negative values were raised by an adaptively high value of aggregation parameter according to Formula (10) and the overestimated $P_{v}$ was simultaneously reduced to fit to the high cross-polarization term [14]. Though both ADAM and 7CD could cut down the negative powers in $P_{s}$ and $P_{d}$, there are some differences between them. ADAM utilizes an adaptive approach to decrease the estimation of $P_{v}$, but 7CD does so by subtracting the asymmetric scattering terms from the volume scattering power, leading to a high possibility of negative powers in $P_{v}$ [16]. According to the principle of ADAM, it can be inferred that a high value of the aggregation parameter 
responding to a target with a high cross-scattering property would result in a high value of $T_{33}$ in a coherency matrix. The high cross-scattering property used in PolSAR images means that an observed target could significantly change the polarization states of radar waves, suggesting that the polarized direction of a radar wave is different than the scatterer's direction [38]. Thus, when the orientation angle of building is around $45^{\circ}$, the scatterer changes the polarization state of vertical wave or horizontal wave to the $45^{\circ}$ direction, and then the recorded cross-polarization term in the PolSAR image will present a high value [38-40].

Table 3. The statistical results of surface scattering power $P_{S}$ in 8 types of samples over San Francisco, USA.

\begin{tabular}{lcccccc}
\hline $\boldsymbol{P}_{\boldsymbol{s}}$ & \multicolumn{3}{c}{ Mean } & \multicolumn{3}{c}{ STD } \\
\cline { 2 - 7 } San Francisco, USA & ADAM & FDD & 7CD & ADAM & FDD & 7CD \\
\hline 20 $0^{\circ}$ oriented building & 0.09 & 0.06 & 0.28 & 0.08 & 0.15 & 0.12 \\
$40^{\circ}$ oriented building & 0.05 & -0.08 & 0.29 & 0.07 & 0.21 & 0.20 \\
$45^{\circ}$ oriented building & 0.07 & 0.04 & 0.21 & 0.06 & 0.11 & 0.13 \\
$50^{\circ}$ oriented building & 0.05 & -0.03 & 0.22 & 0.05 & 0.13 & 0.09 \\
$70^{\circ}$ oriented building & 0.11 & 0.10 & 0.27 & 0.09 & 0.12 & 0.15 \\
$80^{\circ}$ oriented building & 0.12 & 0.21 & 0.34 & 0.08 & 0.13 & 0.17 \\
Forest & 0.04 & 0.02 & 0.10 & 0.03 & 0.05 & 0.05 \\
Water & 0.05 & 0.05 & 0.06 & 0.01 & 0.01 & 0.01 \\
\hline
\end{tabular}

Table 4. The statistical results of double-bounce scattering power $P_{d}$ in 8 types of samples over San Francisco, USA.

\begin{tabular}{lcccccc}
\hline $\boldsymbol{P}_{\boldsymbol{d}}$ & \multicolumn{3}{c}{ Mean } & \multicolumn{3}{c}{ STD } \\
\cline { 2 - 6 } San Francisco, USA & ADAM & FDD & 7CD & ADAM & FDD & 7CD \\
\hline $20^{\circ}$ oriented building & 0.19 & 0.15 & 0.16 & 0.11 & 0.13 & 0.08 \\
$40^{\circ}$ oriented building & 0.20 & 0.02 & 0.13 & 0.16 & 0.23 & 0.10 \\
$45^{\circ}$ oriented building & 0.12 & 0.08 & 0.11 & 0.09 & 0.14 & 0.08 \\
$50^{\circ}$ oriented building & 0.11 & 0.01 & 0.11 & 0.05 & 0.12 & 0.04 \\
$60^{\circ}$ oriented building & 0.18 & 0.18 & 0.17 & 0.12 & 0.13 & 0.10 \\
$70^{\circ}$ oriented building & 0.43 & 0.50 & 0.45 & 0.30 & 0.31 & 0.29 \\
Forest & 0.03 & 0.01 & 0.04 & 0.02 & 0.02 & 0.02 \\
Water & 0.00 & 0.00 & 0.00 & 0.00 & 0.00 & 0.00 \\
\hline
\end{tabular}

Table 5. The statistical results of volume scattering power $P_{v}$ in 8 types of samples over San Francisco, USA.

\begin{tabular}{lcccccc}
\hline $\boldsymbol{P}_{\boldsymbol{v}}$ & \multicolumn{3}{c}{ Mean } & \multicolumn{3}{c}{ STD } \\
\cline { 2 - 7 } San Francisco, USA & ADAM & FDD & 7CD & ADAM & FDD & 7CD \\
\hline $20^{\circ}$ oriented building & 0.43 & 0.50 & -0.02 & 0.14 & 0.18 & 0.18 \\
$40^{\circ}$ oriented building & 0.60 & 0.95 & 0.13 & 0.41 & 0.71 & 0.25 \\
$45^{\circ}$ oriented building & 0.32 & 0.40 & 0.07 & 0.17 & 0.22 & 0.14 \\
$50^{\circ}$ oriented building & 0.38 & 0.58 & 0.21 & 0.14 & 0.25 & 0.17 \\
$60^{\circ}$ oriented building & 0.33 & 0.34 & -0.07 & 0.15 & 0.16 & 0.16 \\
$70^{\circ}$ oriented building & 0.43 & 0.29 & -0.23 & 0.17 & 0.11 & 0.24 \\
Forest & 0.14 & 0.19 & 0.07 & 0.07 & 0.10 & 0.06 \\
Water & 0.02 & 0.01 & -0.01 & 0.00 & 0.00 & 0.00 \\
\hline
\end{tabular}




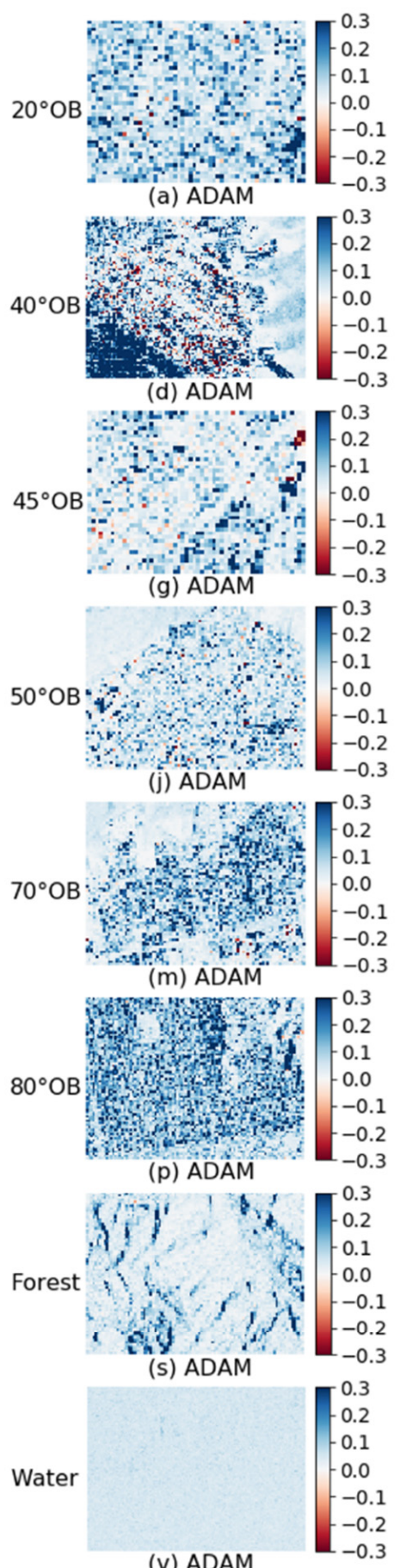

(v) ADAM

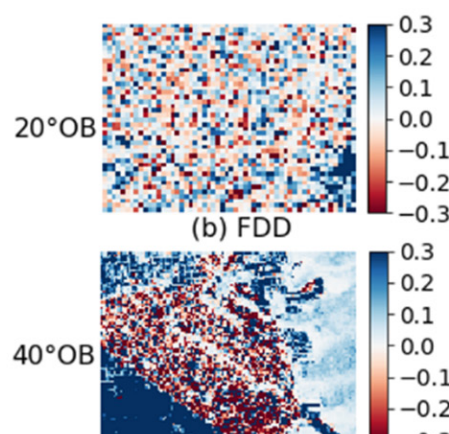

(e) FDD

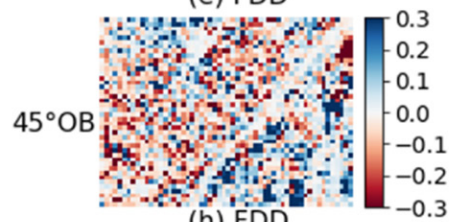

(h) FDD

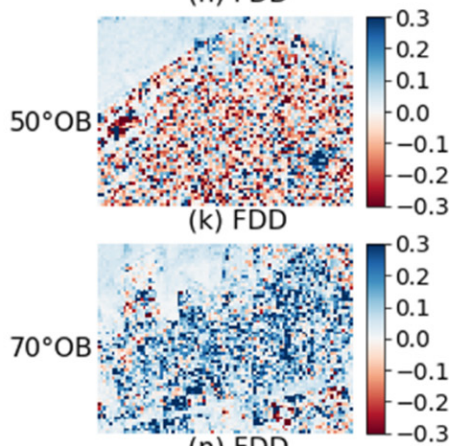

(n) FDD

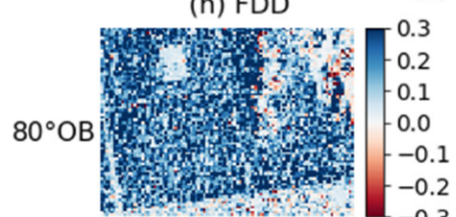

(q) FDD

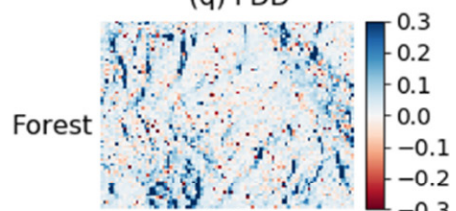

(t) FDD

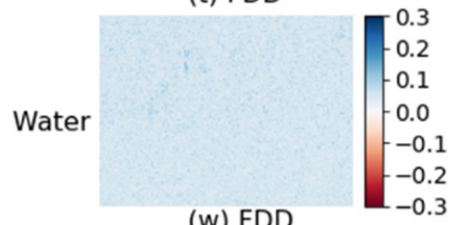

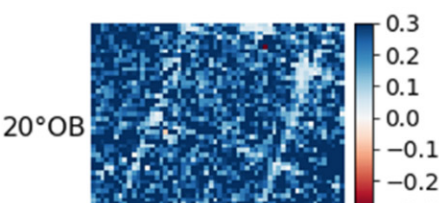

(c) 7CD

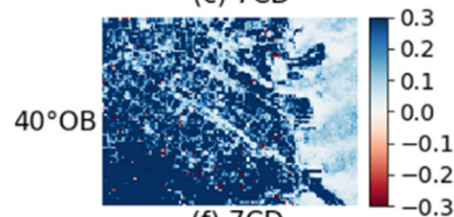

(f) $7 \mathrm{CD}$
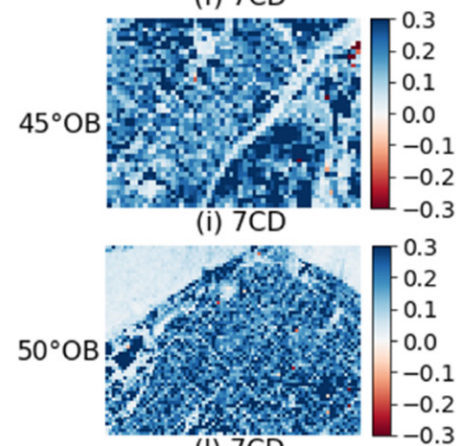

(I) $7 \mathrm{CD}$

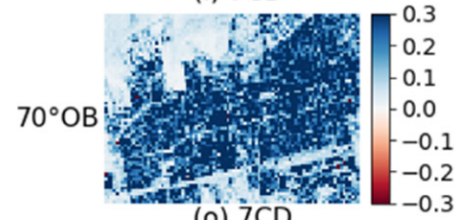

(o) $7 \mathrm{CD}$

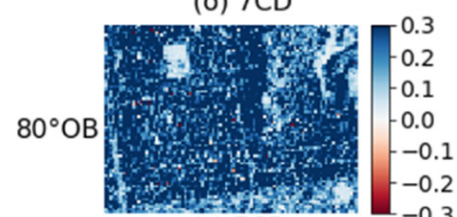

(r) $7 C D$

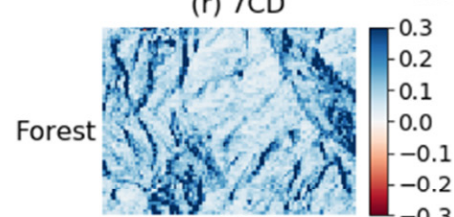

(u) $7 \mathrm{CD}$

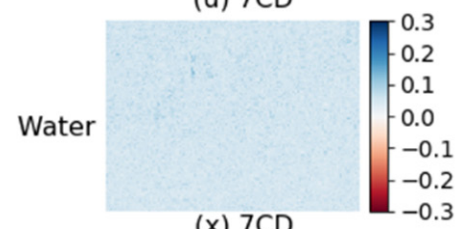

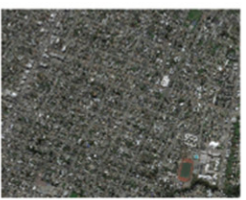
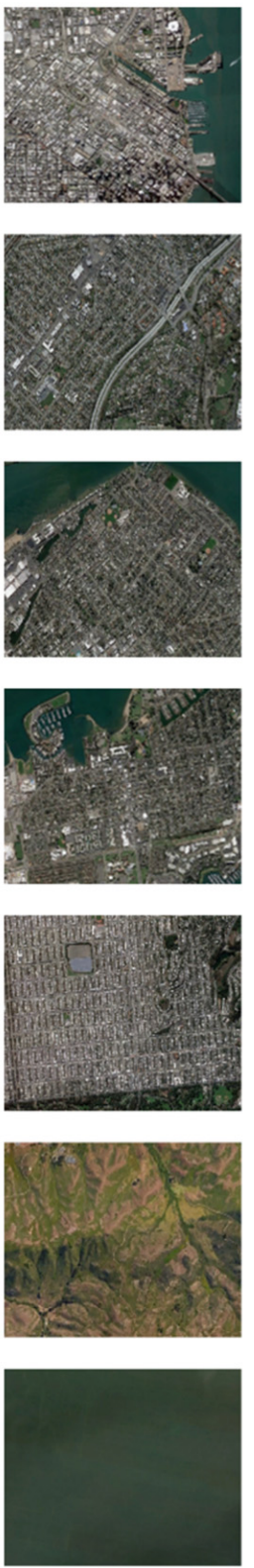

Figure 13. The output map of surface scattering power $P_{S}$ estimated by ADAM, FDD, and 7CD for 8 types of samples over San Francisco, USA. 


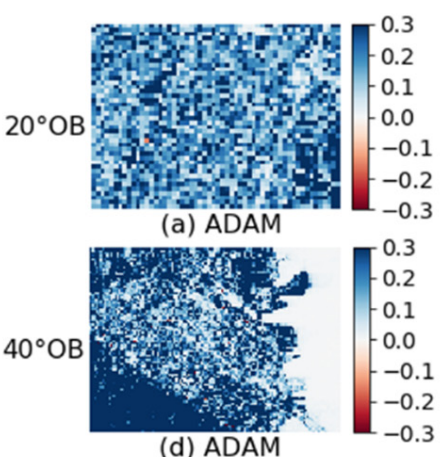

(d) ADAM

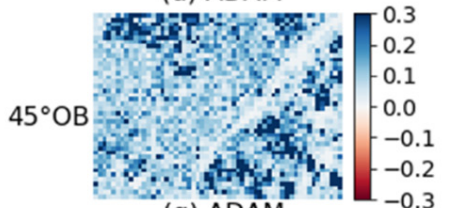

(g) ADAM

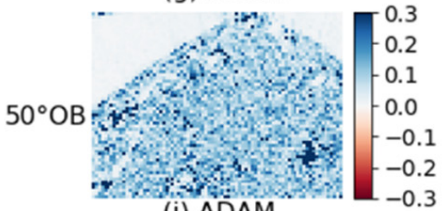

(j) ADAM
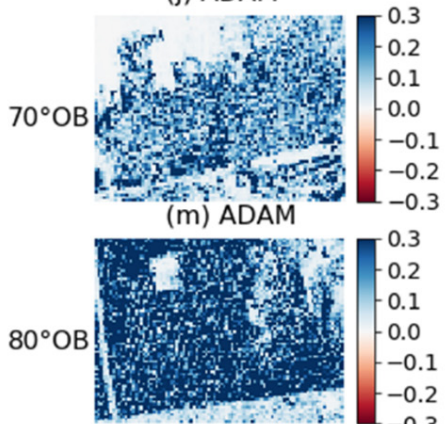

(p) ADAM

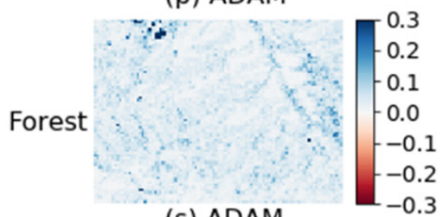

(s) ADAM

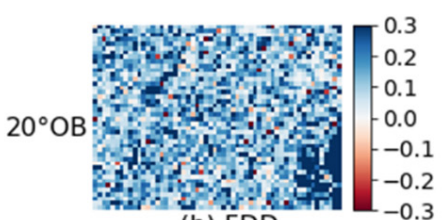

(b) FDD

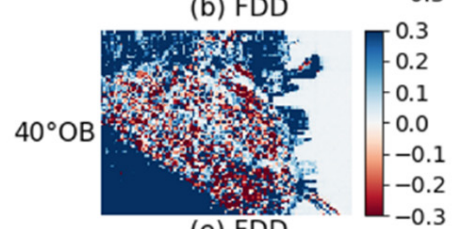

(e) FDD

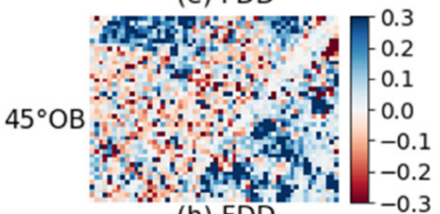

(h) FDD

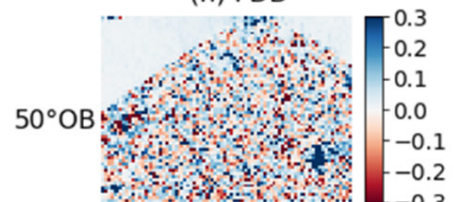

(k) FDD

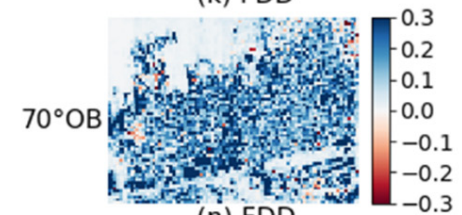

(n) FDD

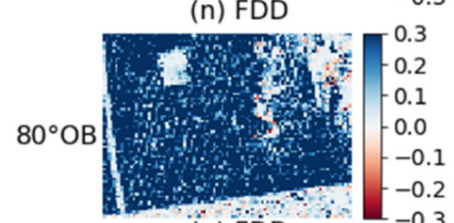

(q) FDD

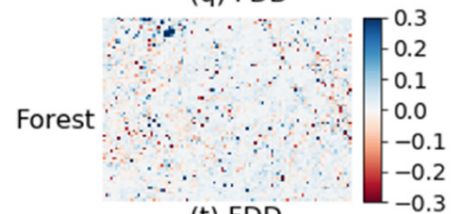

(t) FDD

$\left[\begin{array}{c}0.3 \\ -0.2 \\ -0.1 \\ -0.0 \\ -0.1 \\ -0.2 \\ -0.3\end{array}\right.$

Water

Water

(w) FDD

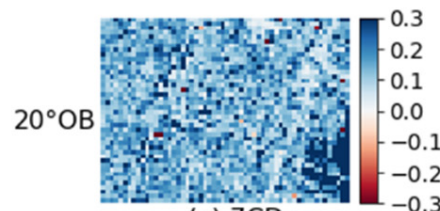

(c) 7CD

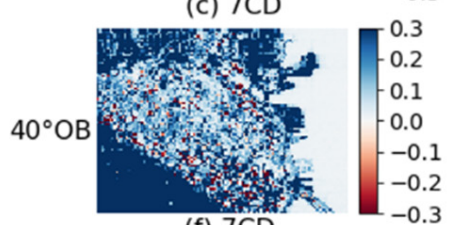

(f) 7CD
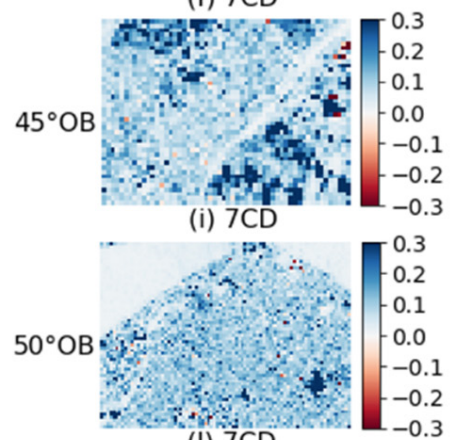

(I) $7 \mathrm{CD}$

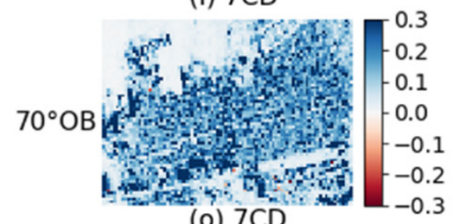

(o) $7 C D$

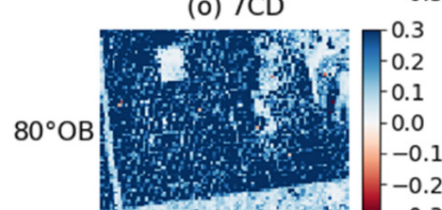

(r) $7 C D$

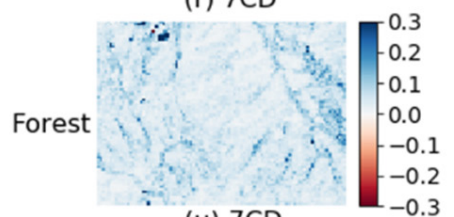

(u) 7CD

$\left[\begin{array}{l}0.3 \\ -0.2 \\ 0.1 \\ -0.0 \\ -0.1 \\ -0.2 \\ -0.3\end{array}\right.$
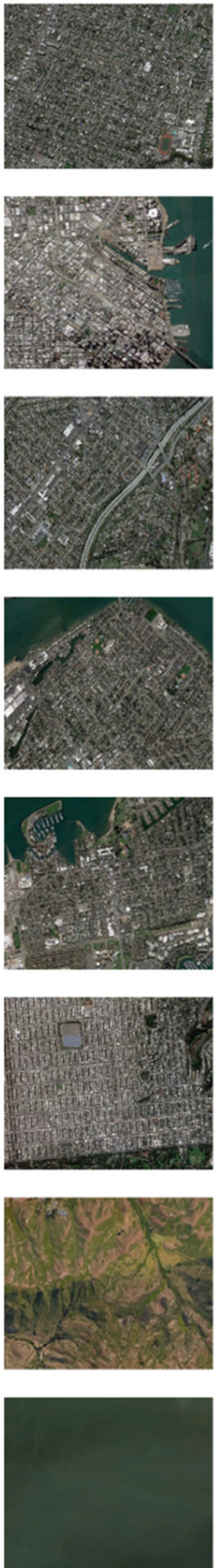

Figure 14. The output map of double-bounce scattering power $P_{d}$ estimated by ADAM, FDD, and 7CD for 8 types of samples over San Francisco, USA. 


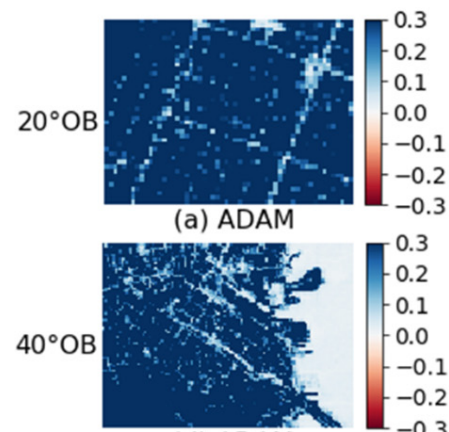

(d) ADAM

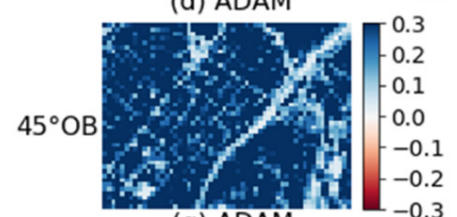

(g) ADAM

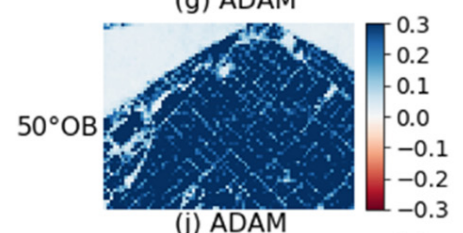

(j) ADAM

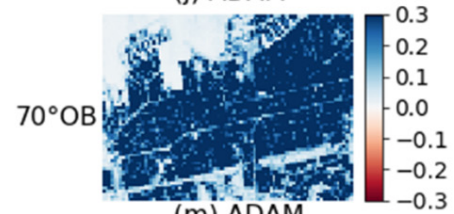

(m) ADAM

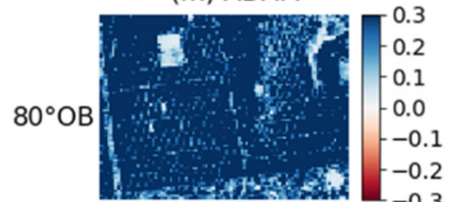

(p) ADAM

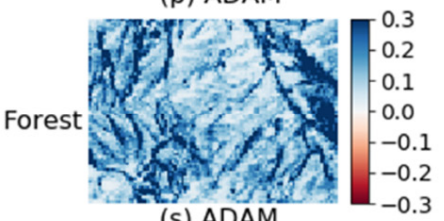

(s) ADAM

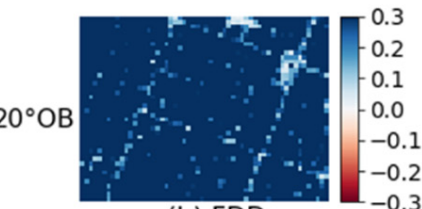

(b) FDD

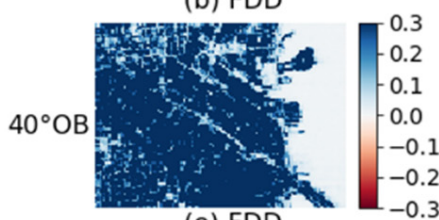

(e) FDD

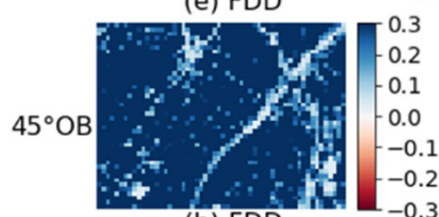

(h) FDD

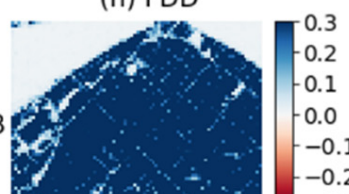

(k) FDD

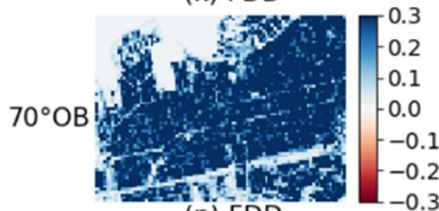

(n) FDD

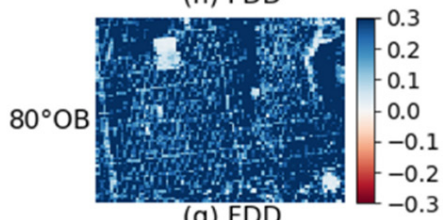

(q) FDD

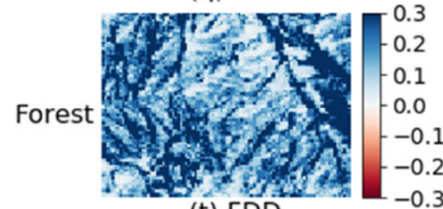

(t) FDD

Water

(w) FDD

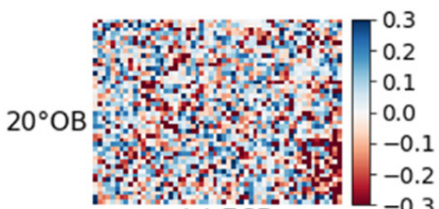

(c) $7 \mathrm{CD}$

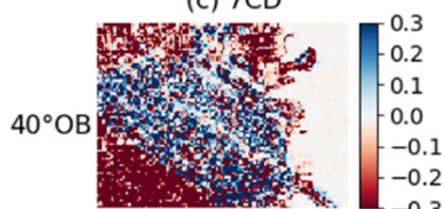

(f) $7 C D$

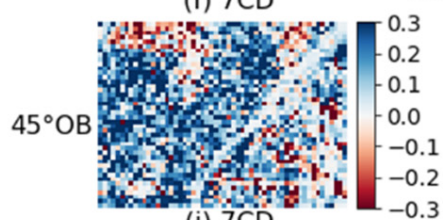

(i) $7 \mathrm{CD}$

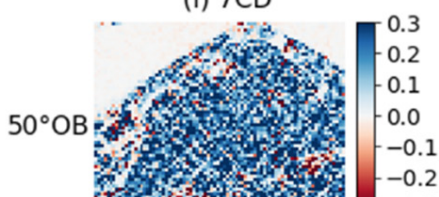

(I) $7 \mathrm{CD}$

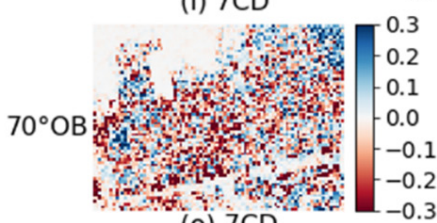

(o) $7 \mathrm{CD}$

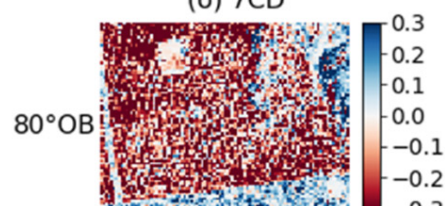

(r) $7 C D$

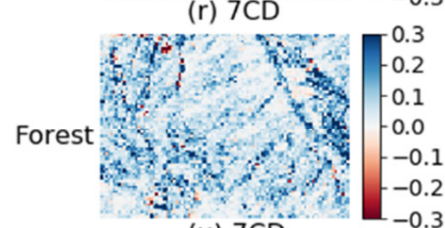

(u) $7 C D$

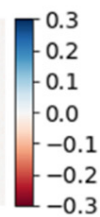

(x) 7CD
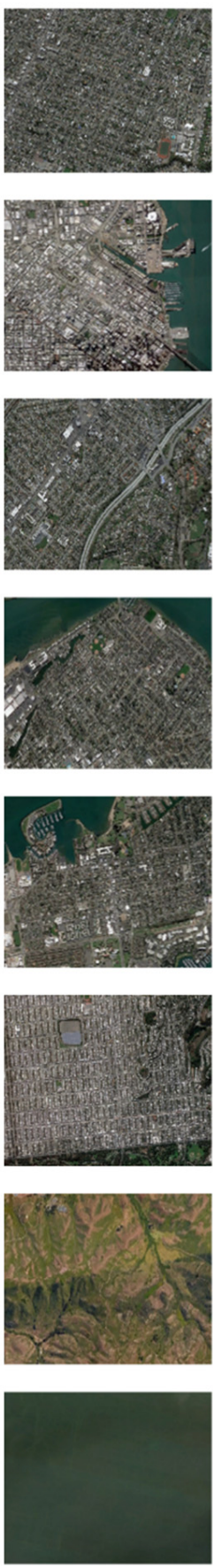

Figure 15. The output map of volume scattering power $P_{v}$ estimated by ADAM, FDD, and 7CD for 8 types of samples over San Francisco, USA.

After comparing the ADAM results of buildings between Figures 13 and 14, we found that the map of surface scattering power had a main color of light blue $(<0.15)$, but the map of double-bounce scattering power was mainly occupied by navy blue $(>0.15)$, which indicated that the contribution of the double-bounce scattering mechanism was higher than that of the surface scattering mechanism. This phenomenon just appeared in the FDD because the orientation angle was equal to $70^{\circ}$, but it presented oppositely in 7CD. The result of ADAM was better fitted because buildings showed higher $P_{d}$ than $P_{s}$ for the dihedral structure formed by the ground-wall in built-up areas predominately contributed 
by double-bounce scattering. Thus, compared with the FDD and 7CD, ADAM was found to be more adaptable in built-up areas.

In terms of forest, ADAM estimated a higher $P_{v}$ value than $P_{d}$ and $P_{s}, 7 \mathrm{CD}$ showed a higher $P_{s}$ value than $P_{v}$ and $P_{d}$, and FDD showed lots of negative powers in the map of $P_{d}$ and $P_{s}$. The result of ADAM was better fitted because the forest showed a higher $P_{v}$ value than $P_{d}$ and $P_{s}$ because the canopy and branches of trees predominately contributed volume scattering [12]. Thus, compared with the FDD and 7CD, ADAM as found to be more adaptable in forest areas. Figures 13-15 show that ADAM, FDD, and 7CD performed similarly over water.

The experiment results in Wuhan, China, are shown in the Supplementary Materials, indicating similar advantages of ADAM to those in the experiments over San Francisco, USA, e.g., a lower possibility of negative powers and a better adaptability for buildings and forests. After comparing the results between San Francisco and Wuhan, we found that ADAM maintained insistence between those two experimental sites for describing the characteristics of the same types of targets.

\section{Discussion}

\subsection{Time Efficiency of ADAM}

For all of the existing methods of adaptive PolSAR decomposition, the first step is to determine the adaptive parameters. Since different approaches can be used to determine adaptive parameters, the computation efficiency of the adaptive method can also perform differently $[15,19-21,23,24,41]$. According to the determination algorithm, the existing model-based decomposition method could be grouped to two categories: the fast group and the time-consuming group [11]. In terms of the fast group, the algorithm has the same number of unknown parameters as the equations, so the adaptive parameter could be directly solved by using equations that are almost as fast as those used in the FDD, and the method's time complexity could be assumed as $O(1)$, as in ADAM or the methods proposed by Cui et al., Chen et al., and Wang et al. [19-21]. The time-consuming group suggests that the number of unknown parameters is more than the involved equations in the model, so solving all the adaptive unknowns there requires that some of them are assigned hypothetical values. Thus, an optimal algorithm with an objective function is used in the time-consuming method to determine which hypothetical value is optimal for each pixel of a PolSAR image. Given that the number of adaptive parameters is $m$ and the number of hypothetical values is $n$, the time complexity of time-consuming methods could be recognized as $O\left(\mathrm{~m}^{n}\right)$, such as in the methods proposed by Arii et al., Chen et al., and Xie et al. $[9,15,17,18]$, the computation efficiency of which is lower than that of the FDD.

\subsection{Simple and Clear Physical Meaning of ADAM}

An important reason that the FDD is widely used is its simplicity, as its outputs correspond to clear physical meaning [11,14]. For instance, the output of the volume scattering component can be explained as the contribution of randomly distributed dipoles [5,12]. However, the improvement of the FDD sometimes requires increasing the complexity and reducing the simplicity of the model, e.g., the approaches proposed by Cui et al., Chen et al., and Wang et al. [19-21]. Freeman improved the adaptability of the FDD by combining the surface scattering model and the double-bounce scattering model [22]. However, the two-component decomposition method sacrifices the clarity of three-component model. For example, the outputs of three-component decomposition could better distinguish open and built-up areas in urban zones through the comparison of surface and double-bounce scattering [42]. In contrast, the proposed ADAM method retains the original mechanism of the three-component model without introducing any other types of scattering models. As shown in Figures 11 and 12, the only parameter introduced by ADAM, the aggregation parameter, also presented a clear physical meaning that responded to the orientation angle of buildings or adaptively fit to the property of vegetation and water. 


\section{Conclusions}

In this paper, a novel ADAM approach was proposed to improve the adaptability of the FDD by using a dipole aggregation model to fit independent volume scattering for each resolution unit of a PolSAR image. The approach was tested with ALOS-2 and Radarsat-2 PolSAR data over San Francisco and Wuhan. The advantages of ADAM were demonstrated in this paper as follows. (1) Compared with the original FDD, it reduces the negative powers and performs better in oriented building areas for fitting to doublebounce scattering, i.e., it is able to show higher $P_{d}$ values than $P_{S}$ values; (2) it has adaptive capability with an aggregation parameter that responds to the scattering characteristics of the target, e.g., the increasing of aggregation parameter leads to more effective scatterers aggregating in the $45^{\circ}$ direction that correspond to a high cross-polarized property that is always presented in $45^{\circ}$ oriented buildings, and when the aggregation parameter is reduced to a low value, the ADAM is adapted to water; and (3) compared with the other adaptive decomposition methods, it had better time efficiency, simplicity and clarity.

Supplementary Materials: The following are available online at https://www.mdpi.com/article/10 $.3390 /$ rs13132583/s1, Figure S1: The determined aggregation parameter $\gamma$ of building samples over Wuhan, China; Figure S2: The output map of surface scattering power Ps estimated by ADAM, FDD and 7CD for 8 types of samples over Wuhan, China; Figure S3: The output map of double-bounce scattering power Pd estimated by ADAM, FDD and 7CD for 8 types of samples over Wuhan, China; Figure S4: The output map of volume scattering power Pv estimated by ADAM, FDD and 7CD for 8 types of samples over Wuhan, China; Table S1: The statistical results of the aggregation parameter $\gamma$ for 8 types of samples over Wuhan, China; Table S2: The statistical results of the Ps in 8 types of samples over Wuhan, China; Table S3: The statistical results of the Pd in 8 types of samples over Wuhan, China; Table S4: The statistical results of the Pv in 8 types of samples over Wuhan, China.

Author Contributions: Z.W. and Q.Z. conceived and designed the study; Z.W. and Q.Z carried out the analysis; Z.W., J.J. and Q.Z. wrote the manuscript; Q.Z. and J.J. collected and managed the utilized data, as well as reviewing the manuscript. All authors have read and agreed to the published version of the manuscript.

Funding: This work was supported jointly by National Natural Science Foundation under Grant No. 41571337, and the National Key Research and Development Program of China under Grant No. 2017YFB0502703.

Acknowledgments: We thank the high-performance computing platform of Peking University for supporting our experiments. Our thanks are also extended to Yanjie Xu for her help in English editing. The ALOS-2 images are provided by JAXA under the ALOS-2 RA-6 Research Project (No. 3319).

Conflicts of Interest: The authors declare no conflict of interest.

\section{References}

1. Ramsey, E., III; Rangoonwala, A.; Suzuoki, Y.; Jones, C.E. Oil detection in a coastal marsh with polarimetric synthetic aperture radar (SAR). Remote Sens. 2011, 3, 2630-2662. [CrossRef]

2. Valcarce-Diñeiro, R.; Arias-Pérez, B.; Lopez-Sanchez, J.M.; Sánchez, N. Multi-temporal dual-and quad-polarimetric synthetic aperture radar data for crop-type mapping. Remote Sens. 2019, 11, 1518. [CrossRef]

3. Bailey, J.; Marino, A. Quad-polarimetric multi-Scale analysis of icebergs in ALOS-2 SAR data: A comparison between icebergs in west and east greenland. Remote Sens. 2020, 12, 1864. [CrossRef]

4. Quan, S.; Xiong, B.; Xiang, D.; Hu, C.; Kuang, G. Scattering characterization of obliquely oriented buildings from PolSAR data using eigenvalue-related model. Remote Sens. 2019, 11, 581. [CrossRef]

5. Freeman, A.; Durden, S.L. Three-component scattering model to describe polarimetric SAR data. In Proceedings of the International Society for Optics and Photonics, San Diego, CA, USA, 12 February 1993.

6. Wiederkehr, N.C.; Gama, F.F.; Castro, P.B.N.; Bispo, P.d.C.; Balzter, H.; Sano, E.E.; Liesenberg, V.; Santos, J.R.; Mura, J.C. Discriminating forest successional stages, forest degradation, and land use in central amazon using alos/palsar-2 full-polarimetric data. Remote Sens. 2020, 12, 3512. [CrossRef]

7. White, L.; McGovern, M.; Hayne, S.; Touzi, R.; Pasher, J.; Duffe, J. Investigating the potential use of radarsat-2 and uas imagery for monitoring the restoration of peatlands. Remote Sens. 2020, 12, 2383. [CrossRef]

8. Omari, K.; Chenier, R.; Touzi, R.; Sagram, M. Investigation of C-band SAR polarimetry for mapping a high-tidal coastal environment in northern Canada. Remote Sens. 2020, 12, 1941. [CrossRef] 
9. Xie, Q.; Ballester-Berman, J.D.; Lopez-Sanchez, J.M.; Zhu, J.; Wang, C. Quantitative analysis of polarimetric model-based decomposition methods. Remote Sens. 2016, 8, 977. [CrossRef]

10. Thompson, A.; Kelly, R. Observations of a coniferous forest at 9.6 and 17.2 ghz: Implications for swe retrievals. Remote Sens. 2019, 11, 6. [CrossRef]

11. Chen, S.-W.; Li, Y.-Z.; Wang, X.-S.; Xiao, S.-P.; Sato, M. Modeling and interpretation of scattering mechanisms in polarimetric synthetic aperture radar: Advances and perspectives. IEEE Signal Process. Mag. 2014, 31, 79-89. [CrossRef]

12. Freeman, A.; Durden, S.L. A three-component scattering model for polarimetric SAR data. IEEE Trans. Geosci. Remote Sens. 1998, 36, 963-973. [CrossRef]

13. van Zyl, J.J.; Arii, M.; Kim, Y. Requirements for model-based polarimetric decompositions. In Proceedings of the 7th European Conference on Synthetic Aperture Radar, Friedrichshafen, Germany, 2-5 June 2008.

14. Lee, J.; Ainsworth, T.L.; Wang, Y. Generalized polarimetric model-based decompositions using incoherent scattering models. IEEE Trans. Geosci. Remote Sens. 2014, 52, 2474-2491. [CrossRef]

15. Arii, M.; van Zyl, J.J.; Kim, Y. Adaptive model-based decomposition of polarimetric SAR covariance matrices. IEEE Trans. Geosci. Remote Sens. 2011, 49, 1104-1113. [CrossRef]

16. Yamaguchi, Y.; Sato, A.; Boerner, W.-M.; Sato, R.; Yamada, H. Four-component scattering power decomposition with rotation of coherency matrix. IEEE Trans. Geosci. Remote Sens. 2011, 49, 2251-2258. [CrossRef]

17. Chen, S.; Wang, X.; Xiao, S.; Sato, M. General polarimetric model-based decomposition for coherency matrix. IEEE Trans. Geosci. Remote Sens. 2014, 52, 1843-1855. [CrossRef]

18. Xie, Q.; Ballester-Berman, J.D.; Lopez-Sanchez, J.M.; Zhu, J.; Wang, C. On the use of generalized volume scattering models for the improvement of general polarimetric model-based decomposition. Remote Sens. 2017, 9, 117. [CrossRef]

19. Cui, Y.; Yamaguchi, Y.; Yang, J.; Park, S.-E.; Kobayashi, H.; Singh, G. Three-component power decomposition for polarimetric SAR data based on adaptive volume scatter modeling. Remote Sens. 2012, 4, 1559-1572. [CrossRef]

20. Chen, S.-W.; Wang, X.-S.; Li, Y.-Z.; Sato, M. Adaptive model-based polarimetric decomposition using PolInSAR coherence. IEEE Trans. Geosci. Remote Sens. 2013, 52, 1705-1718. [CrossRef]

21. Wang, Y.; Yu, W.; Liu, X.; Wang, C.; Kuijper, A.; Guthe, S. Demonstration and analysis of an extended adaptive general four-component decomposition. IEEE J. Sel. Top. Appl. Earth Obs. Remote Sens. 2020, 13, 2573-2586. [CrossRef]

22. Freeman, A. Fitting a two-component scattering model to polarimetric SAR data from forests. IEEE Trans. Geosci. Remote Sens. 2007, 45, 2583-2592. [CrossRef]

23. Li, H.; Li, Q.; Wu, G.; Chen, J.; Liang, S. Adaptive two-component model-based decomposition for polarimetric SAR data without assumption of reflection symmetry. IEEE Trans. Geosci. Remote Sens. 2016, 55, 197-211. [CrossRef]

24. Huang, X.; Wang, J.; Shang, J. An adaptive two-component model-based decomposition on soil moisture estimation for c-band RADARSAT-2 imagery over wheat fields at early growing stages. IEEE Geosci. Remote Sens. Lett. 2016, 13, 414-418. [CrossRef]

25. Cloude, S.R.; Pottier, E. A review of target decomposition theorems in radar polarimetry. IEEE Trans. Geosci. Remote Sens. 1996, 34, 498-518. [CrossRef]

26. Cloude, S.R.; Pottier, E. An entropy based classification scheme for land applications of polarimetric SAR. IEEE Trans. Geosci. Remote Sens. 1997, 35, 68-78. [CrossRef]

27. Yamaguchi, Y.; Moriyama, T.; Ishido, M.; Yamada, H. Four-component scattering model for polarimetric SAR image decomposition. IEEE Trans. Geosci. Remote Sens. 2005, 43, 1699-1706. [CrossRef]

28. Arii, M.; van Zyl, J.J.; Kim, Y. A general characterization for polarimetric scattering from vegetation canopies. IEEE Trans. Geosci. Remote Sens. 2010, 48, 3349-3357. [CrossRef]

29. Wang, Z.; Zeng, Q.; Jiao, J. A new volume scattering model for three-component decomposition of polarimetric SAR data. In Proceedings of the 2018 IEEE International Geoscience and Remote Sensing Symposium, Valencia, Spain, 22-27 July 2018.

30. An, W.; Cui, Y.; Yang, J. Three-component model-based decomposition for polarimetric SAR data. IEEE Trans. Geosci. Remote Sens. 2010, 48, 2732-2739.

31. Rosenqvist, A.; Shimada, M.; Suzuki, S.; Ohgushi, F.; Tadono, T.; Watanabe, M. Operational performance of the ALOS global systematic acquisition strategy and observation plans for ALOS-2 PALSAR-2. Remote Sens. Environ. 2014, 155, 3-12. [CrossRef]

32. Fan, H.; Quan, S.; Dai, D.; Wang, X.; Xiao, S. Refined model-based and feature-driven extraction of buildings from PolSAR images. Remote Sens. 2019, 11, 1379. [CrossRef]

33. Susaki, J.; Kajimoto, M.; Kishimoto, M. Urban density mapping of global megacities from polarimetric SAR images. Remote Sens. Environ. 2014, 155, 334-348. [CrossRef]

34. Duchêne, C.; Bard, S.; Barillot, X.; Ruas, A.; Trevisan, J.; Holzapfel, F. Quantitative and qualitative description of building orientation. In Proceedings of the 7th ICA Workshop on Progress in Automated Map Generalization, Paris, France, 28-30 April 2003.

35. Singh, G.; Malik, R.; Mohanty, S.; Rathore, V.S.; Yamada, K.; Umemura, M.; Yamaguchi, Y. Seven-component scattering power decomposition of POLSAR coherency matrix. IEEE Trans. Geosci. Remote Sens. 2019, 57, 8371-8382. [CrossRef]

36. Lee, J.-S.; Pottier, E. Polarimetric Radar Imaging: From Basics to Applications; CRC Press: New York, NY, USA, 2017.

37. Chen, B.; Wang, S.; Jiao, L.; Stolkin, R.; Liu, H. A three-component fisher-based feature weighting method for supervised PolSAR image classification. IEEE Geosci. Remote Sens. Lett. 2014, 12, 731-735. [CrossRef] 
38. Guinvarc'h, R.; Thirion-Lefevre, L. Cross-polarization amplitudes of obliquely orientated buildings with application to urban Areas. IEEE Geosci. Remote Sens. Lett. 2017, 14, 1913-1917. [CrossRef]

39. Hong, S.-H.; Wdowinski, S. Double-bounce component in cross-polarimetric SAR from a new scattering target decomposition. IEEE Trans. Geosci. Remote Sens. 2013, 52, 3039-3051. [CrossRef]

40. Xiang, D.; Tang, T.; Hu, C.; Fan, Q.; Su, Y. Built-up area extraction from PolSAR imagery with model-based decomposition and polarimetric coherence. Remote Sens. 2016, 8, 685. [CrossRef]

41. Huang, X.; Wang, J.; Shang, J. Simplified adaptive volume scattering model and scattering analysis of crops over agricultural fields using the RADARSAT-2 polarimetric synthetic aperture radar imagery. J. Appl. Remote Sens. 2015, 9, 096026. [CrossRef]

42. Van Zyl, J.J. Unsupervised classification of scattering behavior using radar polarimetry data. IEEE Trans. Geosci. Remote Sens. 1989, 27, 36-45. [CrossRef] 